Tsung-fang Wu*

\title{
On a class of nonlocal nonlinear Schrödinger equations with potential well
}

https://doi.org/10.1515/anona-2020-0020

Received November 10, 2018; accepted February 14, 2019.

Abstract: In this paper we investigate the existence, multiplicity and asymptotic behavior of positive solution for the nonlocal nonlinear Schrödinger equations. We exploiting the relationship between the Nehari manifold and eigenvalue problems to discuss how the Nehari manifold changes as parameters $\mu, \lambda$ changes and show how existence, multiplicity and asymptotic results for positive solutions of the equation are linked to properties of the manifold.

Keywords: Nonlocal nonlinear Schrödinger equations; Nehari manifold; Multiple positive solutions; Concentration-compactness principle

MSC: 35B38, 35B40, 35J20, 35J61

\section{Introduction}

In this paper we are concerned with the existence and multiplicity of positive solutions of the nonlocal nonlinear Schrödinger equation

$$
\left\{\begin{array}{l}
-\Delta u+V_{\mu, \lambda}(x) u+\left(I_{\alpha} \star u^{p}\right)|u|^{p-2} u=f(x)|u|^{2 p-2} u \text { in } \mathbb{R}^{N}, \\
u \in H^{1}\left(\mathbb{R}^{N}\right),
\end{array}\right.
$$

where $N \geq 3, \frac{N+\alpha}{N} \leq p<\frac{N}{N-2}$ and $I_{\alpha}$ is the Riesz potential of order $0<\alpha<\min \left\{N, 2^{\star}\right\}\left(2^{\star}=\frac{2 N}{N-2}\right)$ on the Euclidean space $\mathbb{R}^{N}$, defined for each point $x \in \mathbb{R}^{N} \backslash\{0\}$ by

$$
I_{\alpha}(x)=\frac{\Gamma\left(\frac{N-\alpha}{2}\right)}{\Gamma\left(\frac{\alpha}{2}\right) \pi^{N / 2} 2^{\alpha}|x|^{N-\alpha}}
$$

with $\Gamma$ being the Euler gamma function. Throughout this paper, we assume that the parameters $\mu, \lambda>0$ and the functions $V_{\mu, \lambda}:=\mu g-\lambda a$ and $f$ satisfy the following conditions:

$\left(V_{1}\right) g$ is a nonnegative continuous function on $\mathbb{R}^{N}$;

$\left(V_{2}\right)$ there exists $c>0$ such that the set $\{g<c\}:=\left\{x \in \mathbb{R}^{N} \mid g(x)<c\right\}$ is nonempty and has finite measure; $\left(V_{3}\right) \Omega=\operatorname{int}\left\{x \in \mathbb{R}^{N} \mid g(x)=0\right\}$ is nonempty bounded domain and has a smooth boundary with $\bar{\Omega}=$ $\left\{x \in \mathbb{R}^{N} \mid g(x)=0\right\} ;$

$\left(V_{4}\right) a \in L^{N / 2}\left(\mathbb{R}^{N}\right) \cap L^{\infty}\left(\mathbb{R}^{N}\right)$ and $|\{x \in \bar{\Omega}: a(x)>0\}|>0$

$\left(F_{1}\right) f \in L^{\infty}\left(\mathbb{R}^{N}\right)$ and $|\{x \in \bar{\Omega}: f(x)>0\}|>0$.

Remark 1.1. By condition $\left(V_{4}\right)$, the set $\{x \in \bar{\Omega}: a(x)>0\}$ has positive Lebesgue measure, we can assume that $\lambda_{1}\left(\bar{a}_{\Omega}\right)$ denote the positive principal eigenvalue of the problem

$$
-\Delta u(x)=\lambda \bar{a}_{\Omega}(x) u(x) \text { for } x \in \Omega ; u(x)=0 \text { for } x \in \partial \Omega,
$$

*Corresponding Author: Tsung-fang Wu, Department of Applied Mathematics, National University of Kaohsiung, Kaohsiung 811, Taiwan, E-mail: tfwu@nuk.edu.tw 
where $\bar{a}_{\Omega}$ is a restriction of a on $\bar{\Omega}$ Clearly, $\lambda_{1}\left(\bar{a}_{\Omega}\right)$ has a corresponding positive principal eigenfunction $\phi_{1}$.

In recent years, nonlinear Schrödinger type equation has been widely studied under variant assumptions on potential $g$ and weight function $f$. Most of the literature has focused on the equation for $g$ being a positive potential and $f$ being a positive weight function with satisfies the some assumptions of infinite limits. Moreover, the conditions $\left(V_{1}\right)-\left(V_{3}\right)$ imply that $\mu g$ represents a potential well whose depth is controlled by $\mu . \mu g$ is called a steep potential well if $\mu$ is sufficiently large and one expects to find solutions which localize near its bottom $\Omega$. This problem has found much interest after being first introduced by Bartch and Wang [11] in the study of the existence of positive solutions for nonlinear Schrödinger equations and has been attracting much attention, see $[3,9,10,38,43]$ and the references therein. Later, the steep potential well is introduced to the study of some other types of nonlinear differential equations by some researchers, such as nonlocal nonlinear elliptic equations [18, 24, 33, 34, 45, 46].

When $N=3$ and the nonlocal nonlinear term $\left(I_{\alpha}{ }^{\star} u^{p}\right)|u|^{p-2} u=\left(I^{\star} u^{2}\right) u$ for $\alpha=p=2$. Then Eq. $\left(P_{\mu, \lambda}\right)$ is the one type of the following nonlocal nonlinear Schrödinger equation:

$$
-\Delta u+V(x) u+\sigma\left(I^{\star} u^{2}\right) u=f(x)|u|^{2 p-2} u \text { in } \mathbb{R}^{3},
$$

where $1<p<3$ and the parameter $\sigma>0$. It is easy to know that $u$ is a solution of Eq. (1.2) if and only if $(u, \phi)$ is a solution of the following equation:

$$
\left\{\begin{array}{l}
-\triangle u+V(x) u+\sigma \phi u=f(x)|u|^{2 p-2} u, \text { in } \mathbb{R}^{3}, \\
-\triangle \phi=u^{2}, \text { in } \mathbb{R}^{3} .
\end{array}\right.
$$

It is well known that Eq. (1.3) is called the Schrödinger-Poisson system, which was first introduced in [7] as a physical model describing a charged wave interacting with its own electrostatic field. Eq. (1.3) also appears in the electromagnetic field, semiconductor theory, nonlinear optics and plasma physics. Due to the important applications in physics, Eq. (1.3) has been widely studied via modern variational methods under various hypotheses on the potential function and the nonlinearity; see [2, 4, 16, 23, 30, 31, 35-37] and the references therein. More precisely, Ruiz [30] obtained the existence, nonexistence and multiplicity of radial positive solutions for Eq. (1.3) with $V=f \equiv 1$. It turn out that $p=\frac{3}{2}$ is a critical value for the existence of nontrivial solutions. Ruiz's approach is based on minimizing the energy functional $I$ associated with Eq. (1.3) on a certain manifold that is the Nehari-Pohozaev manifold:

$$
\mathcal{N}_{r}=\left\{u \in H_{r}^{1}\left(\mathbb{R}^{3}\right) \backslash\{0\}: Q(u)=0\right\},
$$

where $H_{r}^{1}\left(\mathbb{R}^{3}\right)$ consists of radially symmetric functions in $H^{1}\left(\mathbb{R}^{3}\right)$ and $Q(u)=0$ is derived by subtracting the Pohozaev identity of equations (1.3) from the equation $2\langle I(u), u\rangle=0$. They proved that when $1<p<3 / 2$ and for $\sigma$ is sufficiently small, Eq. (1.3) has two positive radial solutions $v_{1}, v_{2}$ with

$$
0<I\left(v_{1}\right)<I\left(v_{2}\right)=\inf _{u \in H_{r}^{1}\left(\mathbb{R}^{3}\right)} I(u)<0 .
$$

In recent years, many authors have been studying such topics (existence of two positive solutions which one of the negative energy), for example, Chen [14], Huang et al. [21, 22] and Shen and Han [32], consider the following Schrödinger-Poisson system

$$
\left\{\begin{array}{l}
-\triangle u+(1-\lambda h(x)) u+l(x) \phi u=f(x)|u|^{2 p-2} u, \text { in } \mathbb{R}^{3}, \\
-\triangle \phi=l(x) u^{2}, \text { in } \mathbb{R}^{3},
\end{array}\right.
$$

where $2<p<3, l \in L^{2}\left(\mathbb{R}^{3}\right), f \in C\left(\mathbb{R}^{3}\right)$ changes sign in $\mathbb{R}^{3}$ and $\lim _{|x| \rightarrow \infty} f(x)=f_{\infty}<0$. They proved that system (1.4) has two positive solutions which one of the negative energy for $\lambda>\lambda_{1}(h)$, where $\lambda_{1}(h)$ is the first eigenvalue of $-\Delta+i d$ in $H^{1}\left(\mathbb{R}^{3}\right)$ with nonnegative weight function $h \in L^{3 / 2}\left(\mathbb{R}^{3}\right)$.

Very recently, it has proven in [36] that the problem (1.3) admits a positive solution when $V \equiv 1,1<p \leq$ 2 , and $\sigma$ belongs to a certain interval. To this end, the authors introduced the filtration of the Nehari manifold $\mathcal{N}$, that is

$$
\mathcal{N}(c)=\{u \in \mathcal{N}: I(u)<c\},
$$


and showed that this set $\mathcal{N}(c)$ under the given assumptions is the union of two disjoint nonempty sets, namely,

$$
\mathcal{N}(c)=\mathcal{N}^{(1)} \cup \mathcal{N}^{(2)},
$$

which are both $C^{1}$ sub-manifolds of $\mathcal{N}(c)$ and natural constraints of $I$. Moreover, $\mathcal{N}^{(1)}$ is bounded such that $I$ is coercive and bounded below on it, whereas $I$ is unbounded below on $\mathcal{N}^{(2)}$. Moreover, they use the argument of concentration compactness principle to obtain a minimizer of $I$ on $\mathcal{N}^{(1)}$, which is a critical point of $I$. Actually the authors also established $\mathcal{N}^{(2)}$ may not contain any non-zero critical point of $I$ for $\frac{1+\sqrt{73}}{6}<p \leq 2$.

Motivated by the above works [14, 21, 22, 30, 32, 36], in the present article we mainly study the existence and multiplicity of positive solutions for Eq. $\left(P_{\mu, \lambda}\right)$ can not require conditions $f$ changes sign in $\mathbb{R}^{3}$ and $\lim _{|x| \rightarrow \infty} f(x)=f_{\infty}<0$. Furthermore, the existence of least energy positive solutions with negative energy and asymptotic behavior of positive solutions are also discussed. The main method of this paper is to consider minimization on two distinct components of the Nehari manifold corresponding to Eq. $\left(P_{\mu, \lambda}\right)$. The approach to Eq. $\left(P_{\mu, \lambda}\right)$ has been inspired by the papers of $[12,13,44]$. They used the Nehari manifold and fibrering maps to study the bifurcation phenomena for a nonlinear elliptic problem on bounded domains or $\mathbb{R}^{N}$. Since Eq. $\left(P_{\mu, \lambda}\right)$ is on $\mathbb{R}^{N}$, its variational setting is characterized by lack of compactness. To overcome this difficulty we apply a simplified version of the steep well method of [11] and concentration compactness principle of [27]. Furthermore, the first eigenvalue of problem $-\Delta u+\mu g(x) u=\lambda a(x) u$ in $\mathbb{R}^{N}$ is less than $\lambda_{1}\left(\bar{a}_{\Omega}\right)$, which indicates that the original method at $[12,13,21]$ cannot be directly applied, thus we provide an approximation estimate of eigenvalue to prove that the existence of positive solution for Eq. $\left(P_{\mu, \lambda}\right)$ when $0<\lambda<\lambda_{1}\left(\bar{a}_{\Omega}\right)$.

The first result is to establish the existence of least energy positive solutions and the asymptotic behavior of the solutions for Eq. $\left(P_{\mu, \lambda}\right)$ with $0<\lambda<\lambda_{1}\left(\bar{a}_{\Omega}\right)$.

Theorem 1.1. For any $0<\lambda<\lambda_{1}\left(\bar{a}_{\Omega}\right)$ there exists $\widetilde{\mu}_{0}(\lambda)>0$ with $\lim _{\lambda \rightarrow \lambda_{1}^{-}\left(\bar{a}_{\Omega}\right)} \widetilde{\mu}_{0}(\lambda)=\infty$ such that for every $\mu>\widetilde{\mu}_{0}(\lambda)$, Eq. $\left(P_{\mu, \lambda}\right)$ has a least energy positive solution $u_{\mu, \lambda}$.

Next, we now consider what happens as $\lambda \rightarrow \lambda_{1}^{-}\left(\bar{a}_{\Omega}\right)$ or $\mu \rightarrow \infty$. Let

$$
B(u):=-\int_{\mathbb{R}^{N}}\left(I_{\alpha}{ }^{\star}|u|^{p}\right)|u|^{p} d x+\int_{\mathbb{R}^{N}} f|u|^{2 p} d x .
$$

Then we have the following result.

Theorem 1.2. (i) Suppose that $B\left(\phi_{1}\right)>0$. Let $\lambda_{n} \rightarrow \lambda_{1}^{-}\left(\bar{a}_{\Omega}\right)$ and $\mu_{n}>\widetilde{\mu}_{0}\left(\lambda_{n}\right)$ be as in Theorem 1.1 and let $u_{n}:=u_{\mu_{n}, \lambda_{n}}$ be the least energy positive solution of Eq. $\left(P_{\mu_{n}, \lambda_{n}}\right)$ obtained by Theorem 1.1. Then $u_{n} \rightarrow 0$ in $X$ as $n \rightarrow \infty$.

(ii) For $0<\lambda<\lambda_{1}\left(\bar{a}_{\Omega}\right)$. Let $u_{\mu, \lambda}$ be the least energy positive solution obtained in Theorem 1.1. Then $u_{\mu, \lambda} \rightarrow u_{\lambda}^{\infty}$ in $X$ as $\mu \rightarrow \infty$, where $u_{\lambda}^{\infty} \in H_{0}^{1}(\Omega)$ is a positive solution of

$$
\begin{cases}-\Delta u-\lambda \bar{a}_{\Omega}(x) u+\left(I_{\alpha} \star u^{p}\right)|u|^{p-2} u=f(x)|u|^{2 p-2} u & \text { in } \Omega, \\ u=0, & \text { on } \partial \Omega .\end{cases}
$$

The second result is to establish the existence of multiple positive solutions for Eq. $\left(P_{\mu, \lambda}\right)$ with $\lambda>\lambda_{1}\left(\bar{a}_{\Omega}\right)$.

Theorem 1.3. Suppose that $B\left(\phi_{1}\right)<0$. Then there exists $\delta_{0}>0$ such that for any $\lambda_{1}\left(\bar{a}_{\Omega}\right)<\lambda<\lambda_{1}\left(\bar{a}_{\Omega}\right)+\delta_{0}$ and for $\mu$ enough large, Eq. $\left(P_{\mu, \lambda}\right)$ has two positive solutions $u_{\mu, \lambda}^{(1)}$ and $u_{\mu, \lambda}^{(2)}$ with $u_{\mu, \lambda}^{(1)}$ is negative energy and $u_{\mu, \lambda}^{(2)}$ is positive energy. Furthermore, $u_{\mu, \lambda}^{(1)}$ is the least energy positive solution of $E q .\left(P_{\mu, \lambda}\right)$.

Finally, we investigate the nature of least energy positive solution $u_{\mu, \lambda}^{(1)}$ as $\lambda \rightarrow \lambda_{1}^{+}\left(\bar{a}_{\Omega}\right)$ and $\mu \rightarrow \infty$. As mentioned in the introduction a curve of positive solutions bifurcates to the right at $\lambda_{1}\left(\bar{a}_{\Omega}\right)$ when $B\left(\phi_{1}\right)<0$ and $\mu$ sufficiently large. The following theorem implies that $u_{\mu, \lambda}^{(1)}$ will lie on this branch and the concentration of of the solutions for Eq. $\left(P_{\mu, \lambda}\right)$ with $\lambda>\lambda_{1}\left(\bar{a}_{\Omega}\right)$. 
Theorem 1.4. (i) Suppose that $B\left(\phi_{1}\right)<0$. Let $\lambda_{n} \rightarrow \lambda_{1}^{+}\left(\bar{a}_{\Omega}\right)$ and $\mu_{n} \rightarrow \infty$ be as in Theorem 1.3 and let $u_{n}^{(1)}:=u_{\mu_{n}, \lambda_{n}}^{(1)}$ be the least energy positive solutions of Eq. $\left(P_{\mu_{n}, \lambda_{n}}\right)$ obtained by Theorem 1.3. Then

$$
u_{n} \rightarrow 0 ; \frac{u_{n}}{\left\|u_{n}\right\|_{\mu_{n}}} \rightarrow \phi_{1} \quad \text { in } X \text { as } n \rightarrow \infty .
$$

(ii) For $\lambda_{1}\left(\bar{a}_{\Omega}\right)<\lambda<\lambda_{1}\left(\bar{a}_{\Omega}\right)+\delta_{0}$. Let $u_{\mu, \lambda}^{(j)}(j=1,2)$ be the positive solutions obtained in Theorem 1.3. Then $u_{\mu, \lambda}^{(j)} \rightarrow u_{\lambda}^{(j), \infty}$ in $X$ as $\mu \rightarrow \infty$, where $u_{\lambda}^{(j), \infty} \in H_{0}^{1}(\Omega)$ are positive solutions of Eq. $\left(P_{\infty}\right)$.

Remark 1.2. In fact, our method can also be applied to the Choquard equation involving nonautonomous perturbation:

$$
\left\{\begin{array}{l}
-\Delta u+V_{\mu, \lambda}(x) u=\left(I_{\alpha} \star u^{p}\right)|u|^{p-2} u+f(x)|u|^{2 p-2} u \text { in } \mathbb{R}^{N}, \\
u \in H^{1}\left(\mathbb{R}^{N}\right),
\end{array}\right.
$$

and obtain the same conclusions as all the previous theorems under the same hypotheses and in addition $f$ is change sign in $\bar{\Omega}$. Since the proofs are similarly, and so we leave it to the reader to check. Some progress on the existence of positive solutions to $E q .\left(C_{\mu, \lambda}\right)$, can be refer to [40, 41].

The plan of the paper is as follows. In Section 2, some preliminary results are presented and we discuss the Nehari manifold and examine carefully the connection between the Nehari manifold and the fibrering maps. In Section 3, we discuss the Nehari manifold when $\lambda<\lambda_{1}\left(\bar{a}_{\Omega}\right)$. In particular, we prove that Theorems 1.1, 1.2. In Section 4, we discuss the case when $\lambda>\lambda_{1}\left(\bar{a}_{\Omega}\right)$. In particular, we prove that Theorems 1.3, 1.4.

Throughout this paper we denote a strong convergence by " $\rightarrow$ " and a weak convergence by “ $\rightarrow$ ".

\section{Variational setting and Preliminaries}

In this section, we give the variational setting for Eq. $\left(P_{\mu, \lambda}\right)$. Let

$$
X=\left\{u \in H^{1}\left(\mathbb{R}^{N}\right) \mid \int_{\mathbb{R}^{N}} g u^{2} d x<\infty\right\}
$$

be equipped with the inner product and norm

$$
\langle u, v\rangle=\int_{\mathbb{R}^{N}} \nabla u \nabla v+g u v d x,\|u\|=\langle u, u\rangle^{1 / 2} .
$$

For $\mu>0$, we also need the following inner product and norm

$$
\langle u, v\rangle_{\mu}=\int_{\mathbb{R}^{N}} \nabla u \nabla v+\mu g u v d x,\|u\|_{\mu}=\langle u, u\rangle_{\mu}^{1 / 2} .
$$

It is clear that $\|\cdot\| \leq\|\cdot\|_{\mu}$ for $\mu \geq 1$ and set $X_{\mu}=\left(X,\|\cdot\|_{\mu}\right)$. It follows from conditions $\left(V_{1}\right)$ and $\left(V_{2}\right)$ and the Hölder and Sobolev inequalities that we have

$$
\begin{aligned}
\int_{\mathbb{R}^{N}}\left(|\nabla u|^{2}+u^{2}\right) d x & =\int_{\mathbb{R}^{N}}|\nabla u|^{2} d x+\int_{\{g<c\}} u^{2} d x+\int_{\{g \geq c\}} u^{2} d x \\
& \leq\left(1+|\{g<c\}|^{\frac{2}{N}} S^{-2}\right) \int_{\mathbb{R}^{N}}|\nabla u|^{2} d x+\frac{1}{c} \int_{\mathbb{R}^{N}} g u^{2} d x \\
& \leq \max \left\{1+|\{g<c\}|^{\frac{2}{N}} S^{-2}, \frac{1}{c}\right\} \int_{\mathbb{R}^{N}}\left(|\nabla u|^{2} d x+g u^{2}\right) d x,
\end{aligned}
$$


this implies that the imbedding $X \hookrightarrow H^{1}\left(\mathbb{R}^{N}\right)$ is continuous, where the set $\{g \geq c\}:=\left\{x \in \mathbb{R}^{N} \mid g(x) \geq c\right\}$. Moreover, using conditions $\left(V_{1}\right)$ and $\left(V_{2}\right)$, and the Hölder and Sobolev inequalities again, we have for any $r \in\left[2,2^{*}\right]$,

$$
\begin{aligned}
\int_{\mathbb{R}^{N}}|u|^{r} d x & \leq\left(\int_{\{g \geq c\}} u^{2} d x+\int_{\{g<c\}} u^{2} d x\right)^{\frac{2^{*}-r}{2^{*}-2}}\left(S^{-\frac{2 N}{N-2}}\left(\int_{\mathbb{R}^{N}}|\nabla u|^{2} d x\right)^{\frac{N}{N-2}}\right)^{\frac{r-2}{2^{*}-2}} \\
& \leq\left(\frac{1}{\mu c} \int_{\mathbb{R}^{N}} \mu_{0} g u^{2} d x+|\{g<c\}|^{\frac{2}{N}} S^{-2} \int_{\mathbb{R}^{N}}|\nabla u|^{2} d x\right)^{\frac{2^{*}-r}{2^{*}-2}}\left(S^{-\frac{2 N}{N-2}}\|u\|_{\mu}^{\frac{2 N}{N-2}}\right)^{\frac{r-2}{2^{*}-2}} \\
& \leq \frac{|\{g<c\}|^{\frac{2^{*}-r}{2^{*}}}}{S^{r}}\|u\|_{\mu}^{r} \text { for } \mu \geq \mu_{0}:=S^{2} c^{-1}|\{g<c\}|^{-\frac{2}{N}},
\end{aligned}
$$

where, $S$ the best constant for the embedding of $D^{1,2}\left(\mathbb{R}^{N}\right)$ in $L^{2^{*}}\left(\mathbb{R}^{N}\right)$. Moreover, if we assume that $u \in$ $L^{\frac{2 N p}{N+\alpha}}\left(\mathbb{R}^{N}\right)$, then by the Hardy-Littlewood-Sobolev inequality ( see $[20,25,26]$ ) to the function $|u|^{p} \in$ $L^{\frac{2 N}{N+\alpha}}\left(\mathbb{R}^{N}\right)$, we obtain, in view of the Hölder inequality and (2.1),

$$
\begin{aligned}
\int_{\mathbb{R}^{N}}\left(I_{\alpha}{ }^{*}|u|^{p}\right)|u|^{p} d x & \leq\left(\left.\left.\int_{\mathbb{R}^{N}}\left|I_{\alpha}{ }^{*}\right| u\right|^{p}\right|^{\frac{2 N}{N-\alpha}} d x\right)^{\frac{N-\alpha}{2 N}}\left(\int_{\mathbb{R}^{N}}|u|^{\frac{2 N p}{N+\alpha}} d x\right)^{\frac{N+\alpha}{2 N}} \\
& \leq C_{N, \alpha, \frac{2 N}{N+\alpha}}\left(\int_{\mathbb{R}^{N}}|u|^{\frac{2 N p}{N+\alpha}} d x\right)^{\frac{N+\alpha}{N}} \\
& \leq \frac{C_{N, \alpha, \frac{2 N}{N+\alpha}}|\{g<c\}|^{1-\frac{p(N-2)}{N+\alpha}}}{S^{\frac{2 N p}{N+\alpha}}}\|u\|_{\mu}^{2 p}
\end{aligned}
$$

where

$$
C_{N, \alpha, \frac{2 N}{N+\alpha}}=\frac{\Gamma\left(\frac{N-\alpha}{2}\right)}{2^{\alpha} \pi^{\alpha / 2} \Gamma\left(\frac{N+\alpha}{2}\right)}\left(\frac{\Gamma\left(\frac{N}{2}\right)}{\Gamma(N)}\right)^{\alpha / N} .
$$

We use the variational methods to find positive solutions of Eq. $\left(P_{\mu, \lambda}\right)$. Associated with the Eq. $\left(P_{\mu, \lambda}\right)$, we consider the energy functional $J_{\mu, \lambda}: X \rightarrow \mathbb{R}^{N}$

$$
J_{\mu, \lambda}(u)=\frac{1}{2} A_{\mu, \lambda}(u)-\frac{1}{2 p} B(u),
$$

where

$$
A_{\mu, \lambda}(u):=\|u\|_{\mu}^{2}-\lambda \int_{\mathbb{R}^{N}} a u^{2} d x
$$

and

$$
B(u):=-\int_{\mathbb{R}^{N}}\left(I_{\alpha}{ }^{\star}|u|^{p}\right)|u|^{p} d x+\int_{\mathbb{R}^{N}} f|u|^{2 p} d x .
$$

Because the energy functional $J_{\mu, \lambda}$ is not bounded below on $X$, it is useful to consider the functional on the Nehari manifold (see [29])

$$
\mathbf{N}_{\mu, \lambda}=\left\{u \in X \backslash\{0\} \mid\left\langle J_{\mu, \lambda}^{\prime}(u), u\right\rangle=0\right\} .
$$

Thus, $u \in \mathbf{N}_{\mu, \lambda}$ if and only if

$$
A_{\mu, \lambda}(u)-B(u)=0 .
$$

Hence, if $u \in \mathbf{N}_{\mu, \lambda}$, then

$$
J_{\mu, \lambda}(u)=\left(\frac{1}{2}-\frac{1}{2 p}\right) A_{\mu, \lambda}(u)=\left(\frac{1}{2}-\frac{1}{2 p}\right) B(u)
$$


Note that $\mathbf{N}_{\mu, \lambda}$ contains every nonzero solution of Eq. $\left(P_{\mu, \lambda}\right)$. It is useful to understand $\mathbf{N}_{\mu, \lambda}$ in terms of the stationary points of mappings of the form $h_{u}(t)=J_{\mu, \lambda}(t u)(t>0)$. Such a map is known as the fibrering map. It was introduced by Drábek and Pohozaev [17], and further discussed by Brown and Zhang [12]. It is clear that, if $u$ is a local minimizer of $J_{\mu, \lambda}$, then $h_{u}$ has a local minimum at $t=1$. Moreover, $t u \in \mathbf{N}_{\mu, \lambda}$ if and only if $h_{u}^{\prime}(t)=0$ for $u \in X \backslash\{0\}$. Thus, points in $\mathbf{N}_{\mu, \lambda}$ correspond to stationary points of the maps $h_{u}$ and so it is natural to divide $\mathbf{N}_{\mu, \lambda}$ into three subsets $\mathbf{N}_{\mu, \lambda}^{+}, \mathbf{N}_{\mu, \lambda}^{-}$and $\mathbf{N}_{\mu, \lambda}^{0}$ corresponding to local minima, local maxima and points of inflexion of fibrering maps. We have

$$
h_{u}^{\prime}(t)=t A_{\mu, \lambda}(u)-t^{2 p-1} B(u)
$$

and

$$
h_{u}^{\prime \prime}(t)=A_{\mu, \lambda}(u)-(2 p-1) t^{2 p-2} B(u) .
$$

Hence if we define

$$
\begin{aligned}
& \mathbf{N}_{\mu, \lambda}^{+}=\left\{u \in \mathbf{N}_{\mu, \lambda}: A_{\mu, \lambda}(u)-(2 p-1) B(u)>0\right\} ; \\
& \mathbf{N}_{\mu, \lambda}^{0}=\left\{u \in \mathbf{N}_{\mu, \lambda}: A_{\mu, \lambda}(u)-(2 p-1) B(u)=0\right\} ; \\
& \mathbf{N}_{\mu, \lambda}^{-}=\left\{u \in \mathbf{N}_{\mu, \lambda}: A_{\mu, \lambda}(u)-(2 p-1) B(u)<0\right\},
\end{aligned}
$$

which indicates that for $u \in \mathbf{N}_{\mu, \lambda}$, we have $h_{u}^{\prime}(1)=0$ and $u \in \mathbf{N}_{\mu, \lambda}^{+}, \mathbf{N}_{\mu, \lambda}^{0}, \mathbf{N}_{\mu, \lambda}^{-}$if $h_{u}^{\prime \prime}(1)>0, h_{u}^{\prime \prime}(1)=0, h_{u}^{\prime \prime}(1)<$ 0 , respectively. Moreover, it is easy to show that

$$
\begin{aligned}
\mathbf{N}_{\mu, \lambda}^{+} & =\left\{u \in \mathbf{N}_{\mu, \lambda}: A_{\mu, \lambda}(u)-(2 p-1) B(u)>0\right\} \\
& =\left\{u \in \mathbf{N}_{\mu, \lambda}:(2 p-2) B(u)<0\right\} \\
& =\left\{u \in \mathbf{N}_{\mu, \lambda}: B(u)<0\right\} .
\end{aligned}
$$

Similarly,

$$
\mathbf{N}_{\mu, \lambda}^{-}=\left\{u \in \mathbf{N}_{\mu, \lambda}: B(u)>0\right\}
$$

and

$$
\mathbf{N}_{\mu, \lambda}^{0}=\left\{u \in \mathbf{N}_{\mu, \lambda}: B(u)=0\right\} .
$$

Moreover, by (2.4), if $A_{\mu, \lambda}(u)$ and $B(u)$ have the same sign, then $h_{u}$ has exactly one turning point at

$$
t(u)=\left[\frac{A_{\mu, \lambda}(u)}{B(u)}\right]^{\frac{1}{2 p-2}}
$$

and if $A_{\mu, \lambda}(u)$ and $B(u)$ have opposite signs, then $h_{u}$ has no turning points. Thus, if $A_{\mu, \lambda}(u), B(u)>0$, then $h_{u}(t)>0$ for $t$ small and positive but $h_{u}(t) \rightarrow-\infty$ as $t \rightarrow \infty$; also $h_{u}(t)$ has a unique (maximum) stationary point at $t(u)$ and $t(u) u \in \mathbf{N}_{\mu, \lambda}^{-}$. Similarly, if $A_{\mu, \lambda}(u), B(u)<0, h_{u}(t)<0$ for $t$ small and positive, $h_{u}(t) \rightarrow \infty$ as $t \rightarrow \infty$ and $h_{u}(t)$ has a unique minimum at $t(u)$ so that $t(u) u \in \mathbf{N}_{\mu, \lambda}^{+}$. Finally, if $A_{\mu, \lambda}(u) B(u)<0, h_{u}$ is strictly increasing (resp. decreasing) for all $t>0$. Thus, we have the following results.

Lemma 2.1. If $u \in X \backslash\{0\}$, then

(i) a multiple of $u$ lies is $\mathbf{N}_{\mu, \lambda}^{-}$if and only if $A_{\mu, \lambda}(u), B(u)>0$;

(ii) a multiple of $u$ lies is $\mathbf{N}_{\mu, \lambda}^{+}$if and only if $A_{\mu, \lambda}(u), B(u)<0$;

(iii) when $A_{\mu, \lambda}(u) B(u)<0$, no multiple of $u$ lies in $\mathbf{N}_{\mu, \lambda}$.

The following Lemma shows that minimizers on $\mathbf{N}_{\mu, \lambda}$ are critical points for $J_{\mu, \lambda}$ in $X$.

Lemma 2.2. Suppose that $u_{0}$ is a local minimizer for $J_{\mu, \lambda}$ on $\mathbf{N}_{\mu, \lambda}$ and that $u_{0} \notin \mathbf{N}_{\mu, \lambda}^{0}$. Then $J_{\mu, \lambda}^{\prime}\left(u_{0}\right)=0$.

Proof. The proof of Lemma 2.2 is essentially same as that in Brown and Zhang [12, Theorem 2.3] (or see Binding et al. [5]), so we omit it here. 
In order to prove main results, we will use a special case of the classical Brezis-Lieb lemma [8] for Riesz potentials.

Lemma 2.3. (Brezis-Lieb lemma for the Riesz potential [28, Lemma 2.4]). Let $\left\{u_{n}\right\}$ be a bounded sequence in $L^{2}\left(\mathbb{R}^{N}\right)$. If $u_{n} \rightarrow$ u a.e. in $\mathbb{R}^{N}$, then

$$
\int_{\mathbb{R}^{N}}\left(I_{\alpha}{ }^{\star}\left|u_{n}-u\right|^{p}\right)\left|u_{n}-u\right|^{p} d x=\int_{\mathbb{R}^{N}}\left(I_{\alpha}{ }^{*}\left|u_{n}\right|^{p}\right)\left|u_{n}\right|^{p} d x-\int_{\mathbb{R}^{N}}\left(I_{\alpha}{ }^{\star}|u|^{p}\right)|u|^{p} d x+o(1) .
$$

We need the following result.

Lemma 2.4. Let $\mu_{n} \rightarrow \infty$ as $n \rightarrow \infty$ and $\left\{v_{n}\right\} \subset X$ with $\left\|v_{n}\right\|_{\mu_{n}} \leq c_{0}$ for some $c_{0}>0$. Then there exist subsequence $\left\{v_{n}\right\}$ and $v_{0} \in H_{0}^{1}(\Omega)$ such that $v_{n} \rightarrow v_{0}$ in $X$ and $v_{n} \rightarrow v_{0}$ in $L^{r}\left(\mathbb{R}^{N}\right)$ for all $2 \leq r<2^{\star}$ and $B\left(v_{n}\right) \rightarrow B\left(v_{0}\right)$.

Proof. Since $\left\|v_{n}\right\| \leq\left\|v_{n}\right\|_{\mu_{n}} \leq c_{0}$. We may assume that there exists $v_{0} \in X$ such that

$$
\begin{aligned}
& v_{n} \rightarrow v_{0} \text { in } X, \\
& v_{n} \rightarrow v_{0} \text { a.e. in } \mathbb{R}^{N}, \\
& v_{n} \rightarrow v_{0} \text { in } L_{\text {loc }}^{r}\left(\mathbb{R}^{N}\right) \text { for } 2 \leq r<2^{*} .
\end{aligned}
$$

By Fatou's Lemma, we have

$$
\int_{\mathbb{R}^{N}} g v_{0}^{2} d x \leq \liminf _{n \rightarrow \infty} \int_{\mathbb{R}^{N}} g v_{n}^{2} d x \leq \liminf _{n \rightarrow \infty} \frac{\left\|v_{n}\right\|_{\mu_{n}}^{2}}{\mu_{n}}=0,
$$

this implies that $\int_{\mathbb{R}^{N}} g v_{0}^{2} d x=0$ or $v_{0}=0$ a.e. in $\mathbb{R}^{N} \backslash \bar{\Omega}$ and $v_{0} \in H_{0}^{1}(\Omega)$ by condition $\left(V_{3}\right)$. We now show that $v_{n} \rightarrow v_{0}$ in $L^{r}\left(\mathbb{R}^{N}\right)$. Suppose on the contrary. Then by Lions vanishing lemma (see [27, Lemma I.1] or [42, Lemma 1.21]), there exist $d_{0}>0, R_{0}>0$ and $x_{n} \in \mathbb{R}^{N}$ such that

$$
\int_{B^{N}\left(x_{n}, R_{0}\right)}\left(v_{n}-v_{0}\right)^{2} d x \geq d_{0} \text {. }
$$

Moreover, $x_{n} \rightarrow \infty$, and hence, $\left|B\left(x_{n}, R_{0}\right) \cap\left\{x \in \mathbb{R}^{N}: g<c\right\}\right| \rightarrow 0$. By the Hölder inequality, we have

$$
\int_{B\left(x_{n}, R_{0}\right) \cap\{g<c\}}\left(v_{n}-v_{0}\right)^{2} d x \rightarrow 0 .
$$

Consequently,

$$
\begin{aligned}
c_{0} & \geq\left\|v_{n}\right\|_{\mu_{n}}^{2} \geq \mu_{n} c \int_{B\left(x_{n}, R_{0}\right) \cap\{g \geq c\}} v_{n}^{2} d x=\mu_{n} c \int_{B\left(x_{n}, R_{0}\right) \cap\{g \geq c\}}\left(v_{n}-v_{0}\right)^{2} d x \\
& =\mu_{n} c\left(\int_{B\left(x_{n}, R_{0}\right)}\left(v_{n}-v_{0}\right)^{2} d x-\int_{B\left(x_{n}, R_{0}\right) \cap\{g<c\}}\left(v_{n}-v_{0}\right)^{2} d x+o(1)\right) \\
& \rightarrow \infty,
\end{aligned}
$$

which a contradiction. Thus, $v_{n} \rightarrow v_{0}$ in $L^{r}\left(\mathbb{R}^{N}\right)$ for all $2 \leq r<2^{\star}$. Moreover, by (2.2) and Lemma 2.3, $B\left(v_{n}\right) \rightarrow B\left(v_{0}\right)$, since $2 \leq \frac{2 N p}{N+\alpha}<2^{*}$. This completes the proof.

Next, we consider the following eigenvalue problem

$$
-\Delta u(x)+\mu g(x) u(x)=\lambda a(x) u(x) \text { for } x \in \mathbb{R}^{N} .
$$


We can approach this problem by a direct method and attempt to obtain nontrivial solutions of problem (2.6) as relative minima of the functional

$$
I_{\mu}(u)=\frac{1}{2} \int_{\mathbb{R}^{N}}|\nabla u|^{2}+\mu g u^{2} d x,
$$

on the unit sphere in $\mathbb{B}=\left\{u \in X: \int_{\mathbb{R}^{N}} a u^{2} d x=1\right\}$. Equivalently, we may seek to minimize a quotient as follows

$$
\tilde{\lambda}_{1, \mu}(a)=\inf _{u \in X \backslash\{0\}} \frac{\int_{\mathbb{R}^{N}}|\nabla u|^{2}+\mu g u^{2} d x}{\int_{\mathbb{R}^{N}} a u^{2} d x} .
$$

Then, by (2.1),

$$
\frac{\int_{\mathbb{R}^{N}}|\nabla u|^{2}+\mu g u^{2} d x}{\int_{\mathbb{R}^{N}} a u^{2} d x} \geq \frac{S^{2}}{\|a\|_{\infty}|\{g<c\}|^{\frac{2}{N}}} \text { for all } \mu \geq \mu_{0},
$$

this implies that $\widetilde{\lambda}_{1, \mu}(a) \geq \frac{S^{2}}{\|a\|_{\infty}|\{g<c\}|^{\frac{2}{N}}}>0$. Moreover, by condition $\left(V_{3}\right)$,

$$
\inf _{u \in X \backslash\{0\}} \frac{\int_{\mathbb{R}^{N}}|\nabla u|^{2}+\mu g u^{2} d x}{\int_{\mathbb{R}^{N}} a u^{2} d x} \leq \inf _{u \in H_{0}^{1}(\Omega) \backslash\{0\}} \frac{\int_{\mathbb{R}^{N}}|\nabla u|^{2}+\mu g u^{2} d x}{\int_{\mathbb{R}^{N}} a u^{2} d x}=\inf _{u \in H_{0}^{1}(\Omega) \backslash\{0\}} \frac{\int_{\Omega}|\nabla u|^{2}}{\int_{\Omega} \bar{a}_{\Omega} u^{2} d x},
$$

which indicates that $\tilde{\lambda}_{1, \mu}(a) \leq \lambda_{1}\left(\bar{a}_{\Omega}\right)$ for all $\mu>0$. Then we have the following results.

Lemma 2.5. For each $\mu>\mu_{0}$ there exists a positive function $\varphi_{\mu} \in X$ with $\int_{\mathbb{R}^{N}} a \varphi_{\mu}^{2} d x=1$ such that

$$
\tilde{\lambda}_{1, \mu}(a)=\int_{\mathbb{R}^{N}}\left|\nabla \varphi_{\mu}\right|^{2}+\mu g \varphi_{\mu}^{2} d x<\lambda_{1}\left(\bar{a}_{\Omega}\right) .
$$

Furthermore, $\tilde{\lambda}_{1, \mu}(a) \rightarrow \lambda_{1}^{-}\left(\bar{a}_{\Omega}\right)$ and $\varphi_{\mu} \rightarrow \phi_{1}$ as $\mu \rightarrow \infty$, where $\phi_{1}$ is positive principal eigenfunction of problem (1.1).

Proof. Let $\left\{u_{n}\right\} \subset X$ with $\int_{\mathbb{R}^{N}} a u_{n}^{2} d x=1$ be a minimizing sequence of (2.7), that is

$$
\int_{\mathbb{R}^{N}}\left|\nabla u_{n}\right|^{2}+\mu g u_{n}^{2} d x \rightarrow \tilde{\lambda}_{1, \mu}(a) \text { as } n \rightarrow \infty .
$$

Since $\widetilde{\lambda}_{1, \mu}(a) \leq \lambda_{1}\left(\bar{a}_{\Omega}\right)$ for all $\mu \geq 0$, there exists $C_{0}>0$ independent of $\mu$ such that $\left\|u_{n}\right\|_{\mu} \leq C_{0}$. Thus, there exist a subsequence $\left\{u_{n}\right\}$ and $\varphi_{\mu} \in X$ such that

$$
\begin{aligned}
& u_{n} \rightarrow \varphi_{\mu} \text { in } X_{\mu}, \\
& u_{n} \rightarrow \varphi_{\mu} \text { a.e. in } \mathbb{R}^{N}, \\
& u_{n} \rightarrow \varphi_{\mu} \text { in } L_{\text {loc }}^{r}\left(\mathbb{R}^{N}\right) \text { for } 2 \leq r<2^{*} .
\end{aligned}
$$

Moreover, by condition $\left(V_{4}\right)$,

$$
\int_{\mathbb{R}^{N}} a u_{n}^{2} d x \rightarrow \int_{\mathbb{R}^{N}} a \varphi_{\mu}^{2} d x=1 .
$$

Now we show that $u_{n} \rightarrow \varphi_{\mu}$ in $X_{\mu}$. Suppose on the contrary. Then

$$
\int_{\mathbb{R}^{N}}\left|\nabla \varphi_{\mu}\right|^{2}+\mu g \varphi_{\mu}^{2} d x<\liminf _{n \rightarrow \infty} \int_{\mathbb{R}^{N}}\left|\nabla u_{n}\right|^{2}+\mu g u_{n}^{2} d x=\tilde{\lambda}_{1, \mu}(a),
$$

which is impossible. Thus, $u_{n} \rightarrow \varphi_{\mu}$ in $X_{\mu}$, which implies that $\int_{\mathbb{R}^{N}} a \varphi_{\mu}^{2} d x=1$ and $\int_{\mathbb{R}^{N}}\left|\nabla \varphi_{\mu}\right|^{2}+\mu g \varphi_{\mu}^{2} d x=$ $\widetilde{\lambda}_{1, \mu}(a)$. Since $\left|\varphi_{\mu}\right| \in X$ and

$$
\tilde{\lambda}_{1, \mu}(a)=\int_{\mathbb{R}^{N}}\left|\nabla \varphi_{\mu}\right|^{2}+\mu g \varphi_{\mu}^{2} d x=\left.\int_{\mathbb{R}^{N}}|\nabla| \varphi_{\mu}\right|^{2}+\mu g\left|\varphi_{\mu}\right|^{2} d x,
$$


by the maximum principle, we may assume that $\varphi_{\mu}$ is positive eigenfunction of problem $\left(\bar{P}_{\mu}\right)$. Moreover, by the Harnack inequality due to Trudinger [39], we must have $\widetilde{\lambda}_{1, \mu}(a)<\lambda_{1}\left(\bar{a}_{\Omega}\right)$. Now, by the definition of $\widetilde{\lambda}_{1, \mu}(a)$, there holds $\widetilde{\lambda}_{1, \mu_{1}}(a) \leq \widetilde{\lambda}_{1, \mu_{2}}(a)$ for $\mu_{1}<\mu_{2}$. Hence, for any sequence $\mu_{n} \rightarrow \infty$, let $\varphi_{n}:=\varphi_{\mu_{n}}$ be the minimizer of $\lambda_{1, \mu_{n}}(a)$. Then $\int_{\mathbb{R}^{N}} a \varphi_{n}^{2} d x=1$ and

$$
\tilde{\lambda}_{1, \mu_{n}}(a)=\int_{\mathbb{R}^{N}}\left|\nabla \varphi_{n}\right|^{2}+\mu_{n} g \varphi_{n}^{2} d x \leq \lambda_{1}\left(\bar{a}_{\Omega}\right),
$$

that

$$
\tilde{\lambda}_{1, \mu_{n}}(a) \rightarrow d_{0} \leq \lambda_{1}\left(\bar{a}_{\Omega}\right) \text { for some } d_{0}>0
$$

and

$$
\left\|\varphi_{n}\right\| \leq\left\|\varphi_{n}\right\|_{\mu_{n}} \leq \sqrt{\lambda_{1}\left(\bar{a}_{\Omega}\right)}, \text { for } n \text { sufficiently large. }
$$

Thus, by Lemma 2.4, we may assume that there exists $\varphi_{0} \in H_{0}^{1}(\Omega)$ such that $\varphi_{n} \rightarrow \varphi_{0}$ in $X$ and $\varphi_{n} \rightarrow \varphi_{0}$ in $L^{r}\left(\mathbb{R}^{N}\right)$ for all $2 \leq r<2^{*}$. Then

$$
\int_{\Omega}\left|\nabla \varphi_{0}\right|^{2} d x \leq \liminf _{n \rightarrow \infty} \int_{\mathbb{R}^{N}}\left|\nabla \varphi_{n}\right|^{2}+\mu_{n} g \varphi_{n}^{2} d x=d_{0}
$$

and

$$
\lim _{n \rightarrow \infty} \int_{\mathbb{R}^{N}} a \varphi_{n}^{2} d x=\int_{\Omega} \bar{a}_{\Omega} \varphi_{0}^{2} d x=1 .
$$

Since $d_{0} \leq \lambda_{1}\left(\bar{a}_{\Omega}\right)$ and $\lambda_{1}\left(\bar{a}_{\Omega}\right)$ is positive principal eigenvalue of problem (1.1). Thus, we must has $\int_{\Omega}\left|\nabla \varphi_{0}\right|^{2} d x=\lambda_{1}\left(\bar{a}_{\Omega}\right)$ and $\varphi_{0}=\phi_{1}$ a positive principal eigenfunction of problem (1.1), which completes the proof.

\section{The Proof of Theorems 1.1, 1.2 $\left(\lambda<\lambda_{1}\left(\bar{a}_{\Omega}\right)\right)$}

First, we investigate the behavior of $J_{\mu, \lambda}$ on $\mathbf{N}_{\mu, \lambda}^{-}$.

Lemma 3.1. For each $0<\lambda<\lambda_{1}\left(\bar{a}_{\Omega}\right)$ there exists $\bar{\mu}_{0}(\lambda) \geq \mu_{0}$ with $\lim _{\lambda \rightarrow \lambda_{1}^{-}\left(\bar{a}_{\Omega}\right)} \bar{\mu}_{0}(\lambda)=\infty$ such that for every $\mu>\bar{\mu}_{0}(\lambda)$, we have

(i) $\mathbf{N}_{\mu, \lambda}=\mathbf{N}_{\mu, \lambda}^{-}$;

(ii) the energy functional $J_{\mu, \lambda}$ is coercive and bounded below on $\mathbf{N}_{\mu, \lambda}^{-}$. Furthermore, there exists $d_{0}>0$ such that

$$
\inf _{u \in \mathbf{N}_{\mu, \lambda}^{-}} J_{\mu, \lambda}(u) \geq \frac{(p-1)\left(\widetilde{\lambda}_{1, \mu}(a)-\lambda\right)}{2 p \lambda_{1, \mu}(a)} d_{0}^{1 /(p-1)}>0
$$

for all $u \in \mathbf{N}_{\mu, \lambda}^{-}$.

Proof. (i) By Lemma 2.5, for each $0<\lambda<\lambda_{1}\left(\bar{a}_{\Omega}\right)$ there exists $\bar{\mu}_{0}(\lambda) \geq \mu_{0}$ such that for every $\mu>\bar{\mu}_{0}(\lambda)$, there holds $\lambda<\widetilde{\lambda}_{1, \mu}(a) \leq \lambda_{1}\left(\bar{a}_{\Omega}\right)$, which indicates that

$$
A_{\mu, \lambda}(u)=\|u\|_{\mu}^{2}-\lambda \int_{\mathbb{R}^{N}} a u^{2} d x \geq \frac{\widetilde{\lambda}_{1, \mu}(a)-\lambda}{\widetilde{\lambda}_{1, \mu}(a)}\|u\|_{\mu}^{2}>0 \text { for all } u \in X \backslash\{0\} .
$$

Thus, by Lemma 2.1, the submanifolds $\mathbf{N}_{\mu, \lambda}^{+}$and $\mathbf{N}_{\mu, \lambda}^{0}$ are empty and so $\mathbf{N}_{\mu, \lambda}=\mathbf{N}_{\mu, \lambda}^{-}$.

(ii) By (2.1) and (3.2), for each $\mu>\bar{\mu}_{0}(\lambda)$ and $u \in \mathbf{N}_{\mu, \lambda}^{-}$, we obtain

$$
\begin{aligned}
\frac{\widetilde{\lambda}_{1, \mu}(a)-\lambda}{\widetilde{\lambda}_{1, \mu}(a)}\|u\|_{\mu}^{2} & \leq A_{\mu, \lambda}(u)<(2 p-1) B(u) \\
& \leq(2 p-1)\|f\|_{\infty}|\{g<c\}|^{\frac{2^{*}-2 p}{2^{*}}} S^{-2 p}\|u\|_{\mu}^{2 p},
\end{aligned}
$$


which indicates that

$$
\|u\|_{\mu} \geq d_{0}:=\left(\frac{S^{p}\left(\widetilde{\lambda}_{1, \mu}(a)-\lambda\right)}{(2 p-1) \widetilde{\lambda}_{1, \mu}(a)\|f\|_{\infty}|\{g<c\}|^{\frac{2^{*}-2 p}{2^{*}}}}\right)^{1 /(2 p-2)} .
$$

Thus,

$$
J_{\mu, \lambda}(u)=\frac{p-1}{2 p} A_{\mu, \lambda}(u) \geq \frac{(p-1)\left(\widetilde{\lambda}_{1, \mu}(a)-\lambda\right)}{2 p \widetilde{\lambda}_{1, \mu}(a)} d_{0}^{1 /(p-1)}>0,
$$

this implies that the energy functional $J_{\mu, \lambda}$ is coercive and bounded below on $\mathbf{N}_{\mu, \lambda}^{-}$. This completes the proof.

We now show that there exists a minimizer on $\mathbf{N}_{\mu, \lambda}^{-}$which is a critical point of $J_{\mu, \lambda}(u)$ and so a nontrivial solution of Eq. $\left(P_{\mu, \lambda}\right)$. First, we define

$$
c_{\lambda}(\Omega)=\left.\inf _{u \in \mathbf{M}_{\mu, \lambda}(\Omega)} J_{\mu, \lambda}\right|_{H_{0}^{1}(\Omega)}(u),
$$

where

$$
\mathbf{M}_{\mu, \lambda}(\Omega)=\left\{u \in H_{0}^{1}(\Omega):\left\langle\left. J_{\mu, \lambda}^{\prime}\right|_{H_{0}^{1}(\Omega)}(u), u\right\rangle=0\right\} .
$$

Note that

$$
\begin{aligned}
\left.J_{\mu, \lambda}\right|_{H_{0}^{1}(\Omega)}(u)= & \frac{1}{2}\left(\int_{\Omega}|\nabla u|^{2} d x-\int_{\Omega} \lambda \bar{a}_{\Omega} u^{2} d x\right) \\
& -\frac{1}{2 p}\left(-\int_{\Omega}\left(I_{\alpha} \star|u|^{p}\right)|u|^{p} d x+\int_{\Omega} f|u|^{2 p} d x\right),
\end{aligned}
$$

a restriction of $J_{\mu, \lambda}$ on $H_{0}^{1}(\Omega)$, and $c_{\lambda}(\Omega)$ independent of $\mu$. Since $0<\lambda<\lambda_{1}\left(\bar{a}_{\Omega}\right)$, similar to the argument of (3.1), we can conclude that $\left.J_{\mu, \lambda}\right|_{H_{0}^{1}(\Omega)}$ is bounded below on $\mathbf{M}_{\mu, \lambda}(\Omega)$. Moreover, $H_{0}^{1}(\Omega) \subset X_{\mu}$ for all $\mu>0$, one can see that

$$
0<\eta \leq \inf _{u \in \mathbf{N}_{\mu, \lambda}^{-}} J_{\mu, \lambda}(u) \leq c_{\lambda}(\Omega) \text { for all } \mu \geq \mu_{0} .
$$

Taking $D_{0}>c_{\lambda}(\Omega)$. Then we have

$$
0<\eta \leq \inf _{u \in \mathbf{N}_{\mu, \lambda}^{-}} J_{\mu, \lambda}(u) \leq c_{\lambda}(\Omega)<D_{0}
$$

for all $\mu \geq \mu_{0}$. Furthermore, we have the following results.

Theorem 3.2. For each $0<\lambda<\lambda_{1}\left(\bar{a}_{\Omega}\right)$ there exists $\widetilde{\mu}_{0}(\lambda) \geq \bar{\mu}_{0}(\lambda)$ such that $J_{\mu, \lambda}$ has a minimizer on $\mathbf{N}_{\mu, \lambda}^{-}$for all $\mu>\widetilde{\mu}_{0}(\lambda)$.

Proof. By Lemma 3.1 and the Ekeland variational principle [19], for each $\mu>\bar{\mu}_{0}(\lambda)$ there exists a minimizing sequence $\left\{u_{n}\right\} \subset \mathbf{N}_{\mu, \lambda}^{-}$such that

$$
\lim _{n \rightarrow \infty} J_{\mu, \lambda}\left(u_{n}\right)=\inf _{u \in \mathbf{N}_{\mu, \lambda}^{-}} J_{\mu, \lambda}(u)>0 \text { and } J_{\mu, \lambda}^{\prime}\left(u_{n}\right)=o(1) .
$$

Since $\inf _{u \in \mathbf{N}_{\mu, \lambda}^{-}} J_{\mu, \lambda}(u)<D_{0}$, again using Lemma 3.1, there exists $C_{0}>0$ such that $\left\|u_{n}\right\|_{\mu} \leq C_{0}$. Thus, there exist a subsequence $\left\{u_{n}\right\}$ and $u_{0} \in X$ such that $J_{\mu, \lambda}\left(u_{0}\right) \geq 0, J_{\mu, \lambda}^{\prime}\left(u_{0}\right)=0$ and

$$
\begin{aligned}
& u_{n} \rightarrow u_{0} \text { in } X_{\mu}, \\
& u_{n} \rightarrow u_{0} \text { a.e. in } \mathbb{R}^{N}, \\
& u_{n} \rightarrow u_{0} \text { in } L_{\text {loc }}^{r}\left(\mathbb{R}^{N}\right) \text { for } 2 \leq r<2^{*} .
\end{aligned}
$$


Then by condition $\left(V_{4}\right)$,

$$
\lim _{n \rightarrow \infty} \int_{\mathbb{R}^{N}} a u_{n}^{2} d x=\int_{\mathbb{R}^{N}} a u_{0}^{2} d x
$$

Moreover, follows from Brezis-Lieb lemma [8] and Lemma 2.3 obtain that

$$
B\left(u_{n}-u_{0}\right)=B\left(u_{n}\right)-B\left(u_{0}\right)+o(1) .
$$

Now we show that $u_{n} \rightarrow u_{0}$ in $X_{\mu}$. Let $v_{n}=u_{n}-u_{0}$. Then $v_{n} \rightarrow 0$ in $X_{\mu}$. By the Sobolev and GagliardoNirenberg inequalities, for any $\mu>\bar{\mu}_{0}(\lambda)$ we have that

$$
\int_{\mathbb{R}^{N}} v_{n}^{2} d x \leq \frac{1}{\mu c} \int_{\{g \geq c\}} \mu g v_{n}^{2} d x+\int_{\{g<c\}} v_{n}^{2} d x \leq \frac{1}{\mu c}\left\|v_{n}\right\|_{\mu}^{2}+o(1)
$$

and

$$
\begin{aligned}
\int_{\mathbb{R}^{N}}\left|v_{n}\right|^{2 p} d x & \leq C_{0}\left(\frac{1}{\mu c}\left\|v_{n}\right\|_{\mu}^{2}\right)^{\frac{2^{*}-2 p}{2^{*}-2}}\left(\int_{\mathbb{R}^{N}}\left|\nabla v_{n}\right|^{2} d x\right)^{\frac{2^{*}(p-1)}{2^{*}-2}}+o(1) \\
& \leq C_{0}\left(\frac{1}{\mu c}\right)^{\frac{2^{*}-2 p}{2^{*}-2}}\left\|v_{n}\right\|_{\mu}^{2 p}+o(1)
\end{aligned}
$$

or

$$
\int_{\mathbb{R}^{N}}\left|v_{n}\right|^{2 p} d x \leq \Pi_{\mu}\left\|v_{n}\right\|_{\mu}^{2 p}+o(1)
$$

where $\Pi_{\mu}=C_{0}\left(\frac{1}{\mu c}\right)^{\frac{2^{*}-2 p}{2^{*}-2}}$. Thus, using (3.4) - (3.6) gives

$$
J_{\mu, \lambda}\left(v_{n}\right)=J_{\mu, \lambda}\left(u_{n}\right)-J_{\mu, \lambda}\left(u_{0}\right)+o(1) \text { and }\left\langle J_{\mu, \lambda}^{\prime}\left(v_{n}\right), v_{n}\right\rangle=o(1) .
$$

Consequently, by (3.5) , (3.6), (3.8) and Lemma 3.1, one has

$$
\begin{aligned}
D_{0} & \geq \inf _{u \in \mathbf{N}_{\mu, \lambda}^{-}} J_{\mu, \lambda}(u)-J_{\mu, \lambda}\left(u_{0}\right) \geq J_{\mu, \lambda}\left(v_{n}\right)-\frac{1}{2 p}\left\langle J_{\mu, \lambda}^{\prime}\left(v_{n}\right), v_{n}\right\rangle+o(1) \\
& \geq \frac{(p-1)\left(\widetilde{\lambda}_{1, \mu}(a)-\lambda\right)}{2 p \widetilde{\lambda}_{1, \mu}(a)}\left\|v_{n}\right\|_{\mu}^{2}+o(1),
\end{aligned}
$$

which shows that there exists a constant $C_{1}>0$ such that

$$
\left\|v_{n}\right\|_{\mu} \leq C_{1}+o(1) \text { for } \lambda>\bar{\mu}_{0}(\lambda) .
$$

Since $1<\frac{N+\alpha}{N}<p<2_{\alpha}^{\star}$, it follows from (3.5), (3.7) and (3.9) that

$$
\begin{aligned}
o(1) & =\left\langle J_{\mu, \lambda}^{\prime}\left(v_{n}\right), v_{n}\right\rangle \geq\left\|v_{n}\right\|_{\mu}^{2}\left(1-\|f\|_{\infty} \Pi_{\mu}\left\|v_{n}\right\|_{\mu}^{2 p-2}\right)+o(1) \\
& \geq\left\|v_{n}\right\|_{\mu}^{2}\left(1-\|f\|_{\infty} \Pi_{\mu} C_{1}^{2 p-2}\right)+o(1)
\end{aligned}
$$

Notice that $\Pi_{\mu} \rightarrow 0$ as $\mu \rightarrow \infty$. Then by (3.10), there exists $\widetilde{\mu}_{0}(\lambda) \geq \bar{\mu}_{0}(\lambda)$ such that for $\mu>\widetilde{\mu}_{0}(\lambda)$, there holds $v_{n} \rightarrow 0$ in $X_{\mu}$. Hence $u_{n} \rightarrow u_{0}$ in $X_{\mu}$ and so

$$
J_{\mu, \lambda}\left(u_{0}\right)=\lim _{n \rightarrow \infty} J_{\mu, \lambda}\left(u_{n}\right)=\inf _{u \in \mathbf{N}_{\mu, \lambda}^{-}} J_{\mu, \lambda}(u),
$$

which indicates that $u_{0}$ is a minimizer on $\mathbf{N}_{\mu, \lambda}^{-}$. This completes the proof. 
We are now ready to prove Theorem 1.1: By Theorem 3.2, $J_{\mu, \lambda}$ has a minimizer $u_{0}$ on $\mathbf{N}_{\mu, \lambda}^{-}$for all $\mu>\widetilde{\mu}_{0}(\lambda)$. Since $B\left(u_{0}\right)>0$ and $u_{0} \notin \mathbf{N}_{\mu, \lambda}^{0}$, by Lemma 2.2, $u_{0}$ is a critical point of $J_{\mu, \lambda}$. Since $J_{\mu, \lambda}(|u|)=J_{\mu, \lambda}(u)$, then without loss of generality we may assume that $u_{0}$ is positive. This completes the proof.

Next, we now consider what happens as $\lambda \rightarrow \lambda_{1}^{-}\left(\bar{a}_{\Omega}\right)$ or $\mu \rightarrow \infty$. As might be expected from the introduction the sign of $B\left(\phi_{1}\right)$ plays an important role. We conclude the following results by considering the case where $B\left(\phi_{1}\right)>0$.

Theorem 3.3. Suppose that $B\left(\phi_{1}\right)>0$. Then

$$
\lim _{\lambda \rightarrow \lambda_{1}^{-}\left(\bar{a}_{\Omega}\right)} \inf _{u \in \mathbf{N}_{\mu, \lambda}^{-}} J_{\mu, \lambda}(u)=0 .
$$

Proof. We may assume without loss of generality that $\left\|\phi_{1}\right\|_{\mu}=1$. For $0<\lambda<\lambda_{1}\left(\bar{a}_{\Omega}\right)$, we must have that $A_{\mu, \lambda}\left(\phi_{1}\right)>0$, which implies that $A_{\mu, \lambda}\left(\phi_{1}\right), B\left(\phi_{1}\right)>0$ for all $\mu>\bar{\mu}_{0}(\lambda)$. Hence $t\left(\phi_{1}\right) \phi_{1} \in \mathbf{N}_{\mu, \lambda}^{-}$, where

$$
t\left(\phi_{1}\right)=\left(\frac{\int_{\mathbb{R}^{N}}\left(\left|\nabla \phi_{1}\right|^{2} d x-\lambda a \phi_{1}^{2}\right) d x}{B\left(\phi_{1}\right)}\right)^{1 /(2 p-2)}=\left(\frac{\left(\lambda_{1}\left(\bar{a}_{\Omega}\right)-\lambda\right) \int_{\mathbb{R}^{N}} a \phi_{1}^{2} d x}{B\left(\phi_{1}\right)}\right)^{1 /(2 p-2)}>0 .
$$

Thus,

$$
\begin{aligned}
J_{\mu, \lambda}\left(t\left(\phi_{1}\right) \phi_{1}\right) & =\frac{p-2}{2 p} \frac{\left[\left(\lambda_{1}\left(\bar{a}_{\Omega}\right)-\lambda\right) \int_{\mathbb{R}^{N}} a \phi_{1}^{2} d x\right]^{p /(p-1)}}{B\left(\phi_{1}\right)^{1 /(p-1)}} \\
& \rightarrow 0 \text { as } \lambda \rightarrow \lambda_{1}^{-}\left(\bar{a}_{\Omega}\right) .
\end{aligned}
$$

Since $0<\inf _{u \in \mathbf{N}_{\mu, \lambda}^{-}} J_{\mu, \lambda}(u) \leq J_{\mu, \lambda}\left(t\left(\phi_{1}\right) \phi_{1}\right)$, it follows that $\lim _{\lambda \rightarrow \lambda_{1}^{-}(a)} \inf _{u \in \mathbf{N}_{\mu, \lambda}^{-}} J_{\mu, \lambda}(u)=0$. This completes the proof.

Next, we are ready to prove Theorem 1.2: (i) Since $\lambda_{n} \rightarrow \lambda_{1}^{-}\left(\bar{a}_{\Omega}\right)$ and $\widetilde{\mu}_{0}\left(\lambda_{n}\right) \rightarrow \infty$ as $n \rightarrow \infty$, we have $\mu_{n} \rightarrow \infty$ as $n \rightarrow \infty$. Firstly, we show that $\left\{u_{n}\right\}$ is bounded. Suppose on the contrary. Then we may assume without loss of generality that $\left\|u_{n}\right\|_{\mu_{n}} \rightarrow \infty$ as $n \rightarrow \infty$. Let $v_{n}=\frac{u_{n}}{\left\|u_{n}\right\|_{\mu_{n}}}$. Since $\left\|v_{n}\right\|_{\mu_{n}}=1$, by Lemma 2.4, there exist subsequence $\left\{v_{n}\right\}$ and $v_{0} \in H_{0}^{1}(\Omega)$ such that $v_{n} \rightarrow v_{0}$ in $L^{r}\left(\mathbb{R}^{N}\right)$ for $2 \leq r<2^{\star}$ and $B\left(v_{n}\right) \rightarrow B\left(v_{0}\right)$. Hence

$$
\lim _{n \rightarrow \infty} \int_{\mathbb{R}^{N}} a v_{n}^{2} d x=\int_{\mathbb{R}^{N}} a v_{0}^{2} d x
$$

By Theorem 3.3,

$$
J_{\mu_{n}, \lambda_{n}}\left(u_{n}\right)=\frac{p-2}{2 p}\left(\left\|u_{n}\right\|_{\mu_{n}}^{2}-\lambda_{n} \int_{\mathbb{R}^{N}} a u_{n}^{2} d x\right)=\frac{p-2}{2 p} B\left(u_{n}\right) \rightarrow 0 \text { as } n \rightarrow \infty,
$$

dividing by $\left\|u_{n}\right\|_{\mu_{n}}^{2}$ it is easy to see that

$$
\lim _{n \rightarrow \infty}\left(\left\|v_{n}\right\|_{\mu_{n}}^{2}-\lambda_{n} \int_{\mathbb{R}^{N}} a v_{n}^{2} d x\right)=0
$$

and

$$
\lim _{n \rightarrow \infty}\left\|u_{n}\right\|_{\mu_{n}}^{2 p-2} B\left(v_{n}\right)=0
$$

Thus,

$$
\lim _{n \rightarrow \infty} \lambda_{n} \int_{\mathbb{R}^{N}} a v_{n}^{2} d x=\lambda_{1}\left(\bar{a}_{\Omega}\right) \int_{\mathbb{R}^{N}} a v_{0}^{2} d x=1
$$


and

$$
\lim _{n \rightarrow \infty} B\left(v_{n}\right)=B\left(v_{0}\right)=0
$$

Now, we show that

$$
\lim _{n \rightarrow \infty} \int_{\mathbb{R}^{N}}\left|\nabla v_{n}\right|^{2} d x=\int_{\mathbb{R}^{N}}\left|\nabla v_{0}\right|^{2} d x
$$

If not, then we may assume that

$$
0 \leq \int_{\mathbb{R}^{N}}\left(\left|\nabla v_{0}\right|^{2}-\lambda_{1}\left(\bar{a}_{\Omega}\right) a v_{0}^{2}\right) d x<\liminf _{n \rightarrow \infty}\left(\left\|v_{n}\right\|_{\mu_{n}}^{2}-\lambda_{n} \int_{\mathbb{R}^{N}} a v_{n}^{2} d x\right)=0
$$

which is impossible. Thus, we must have

$$
\int_{\Omega}\left(\left|\nabla v_{0}\right|^{2}-\lambda_{1}\left(\bar{a}_{\Omega}\right) a v_{0}^{2}\right) d x=\lim _{n \rightarrow \infty}\left(\left\|v_{n}\right\|_{\mu_{n}}^{2}-\lambda_{n} \int_{\mathbb{R}^{N}} a v_{n}^{2} d x\right)=0,
$$

and so $v_{0}=k \phi_{1}$ for some $k$. Since $B\left(v_{0}\right)=|k|^{2 p} B\left(\phi_{1}\right)=0$ and $B\left(\phi_{1}\right)>0$, it follows that $k=0$. But, as $\int_{\mathbb{R}^{N}} a v_{0}^{2} d x \neq 0$, this is impossible. Hence $\left\{u_{n}\right\}$ is bounded. By Lemma 2.4, we may assume that there exists $u_{0} \in H_{0}^{1}(\Omega)$ such that

$$
\lim _{n \rightarrow \infty} \int_{\mathbb{R}^{N}} a u_{n}^{2} d x=\int_{\mathbb{R}^{N}} a u_{0}^{2} d x \text { and } \lim _{n \rightarrow \infty} B\left(u_{n}\right)=B\left(u_{0}\right) .
$$

Moreover, by Theorem 3.3,

$$
J_{\mu_{n}, \lambda_{n}}\left(u_{n}\right)=\frac{p-1}{2 p} A_{\mu_{n}, \lambda_{n}}\left(u_{n}\right)=\frac{p-2}{2 p} B\left(u_{n}\right) \rightarrow 0 \text { as } n \rightarrow \infty,
$$

which indicates that

$$
\lim _{n \rightarrow \infty} B\left(u_{n}\right)=B\left(u_{0}\right)=0
$$

Since

$$
0 \leq \int_{\mathbb{R}^{N}}\left(\left|\nabla u_{0}\right|^{2}-\lambda_{1}\left(\bar{a}_{\Omega}\right) a u_{0}^{2}\right) d x \leq \liminf _{n \rightarrow \infty} A_{\mu_{n}, \lambda_{n}}\left(u_{n}\right)=0,
$$

and so $u_{0}=k \phi_{1}$ for some $k$. Since $B\left(u_{0}\right)=|k|^{2 p} B\left(\phi_{1}\right)=0$ and $B\left(\phi_{1}\right)>0$, it follows that $k=0$ and $u_{0} \equiv 0$. Therefore, $u_{n} \rightarrow 0$ in $X$.

(ii) Here we follow the argument in [9] (or see [45]) to investigate the concentration for positive solutions of Eq. $\left(P_{\mu, \lambda}\right)$. For any sequence $\mu_{n} \rightarrow \infty$, let $u_{n}:=u_{\mu_{n}, \lambda}$ be the positive solutions of Eq. $\left(P_{\mu_{n}, \lambda}\right)$ obtained in Theorem 1.1. By Lemma 3.1 there exists a positive constants $c_{0}$ and $C_{0}$ are independent of $\mu_{n}$ such that $\left\|u_{n}\right\|_{\mu_{n}} \leq c_{0}$ and $J_{\mu_{n}, \lambda}\left(u_{n}\right) \geq C_{0}$. Therefore, by Lemma 2.4, we may assume that there exists $u_{0} \in H_{0}^{1}(\Omega)$ such that $u_{n} \rightarrow u_{0}$ in $X$ and $u_{n} \rightarrow u_{0}$ in $L^{r}\left(\mathbb{R}^{N}\right)$ for all $2 \leq r<2^{\star}$. Now for any $\varphi \in C_{0}^{\infty}(\Omega)$, because $\left\langle J_{\mu_{n}, \lambda}^{\prime}\left(u_{n}\right), \varphi\right\rangle=0$, it is easy to check that

$$
\int_{\Omega} \nabla u_{0} \nabla \varphi d x=\lambda \int_{\Omega} \bar{a}_{\Omega} u_{0} \varphi d x+\int_{\Omega} f\left|u_{0}\right|^{p-2} u_{0} \varphi d x,
$$

that is, $u_{0}$ is a weak solution of Eq. $\left(P_{\infty}\right)$ by the density of $C_{0}^{\infty}(\Omega)$ in $H_{0}^{1}(\Omega)$. Now, we show that $u_{n} \rightarrow u_{0}$ in $X$. Because $\left\langle J_{\mu_{n}, \lambda}^{\prime}\left(u_{n}\right), u_{n}\right\rangle=\left\langle J_{\mu_{n}, \lambda}^{\prime}\left(u_{n}\right), u_{0}\right\rangle=0$, we have

$$
\left\|u_{n}\right\|_{\mu_{n}}^{2}=\lambda \int_{\mathbb{R}^{N}} a\left(u_{n}\right)^{2} d x+\int_{\mathbb{R}^{N}} f\left|u_{n}\right|^{p} d x
$$

and

$$
\left\langle u_{n}, u_{0}\right\rangle_{\mu_{n}}=\lambda \int_{\mathbb{R}^{N}} a u_{n} u_{0} d x+\int_{\mathbb{R}^{N}} f\left|u_{n}\right|^{p-2} u_{n} u_{0} d x
$$


By (3.11), (3.12) and $u_{n} \rightarrow u_{0}$ in $L^{r}\left(\mathbb{R}^{N}\right)$ for all $2 \leq r<2^{\star}$, we have

$$
\lim _{n \rightarrow \infty}\left\|u_{n}\right\|_{\mu_{n}}^{2}=\lim _{n \rightarrow \infty}\left\langle u_{n}, u_{0}\right\rangle_{\mu_{n}}=\lim _{n \rightarrow \infty}\left\langle u_{n}, u_{0}\right\rangle=\left\|u_{0}\right\|^{2} .
$$

On the other hand, the weakly lower semi-continuity of norm yields

$$
\left\|u_{0}\right\|^{2} \leq \liminf _{n \rightarrow \infty}\left\|u_{n}\right\|^{2} \leq \lim _{n \rightarrow \infty}\left\|u_{n}\right\|_{\mu_{n}}^{2},
$$

and thus, $u_{n} \rightarrow u_{0}$ in $X$. Moreover, by $J_{\mu_{n}, \lambda}\left(u_{n}\right) \geq C_{0}>0$, one has $u_{0} \neq 0$, which completes the proof.

\section{The Proof of Theorems 1.3, $1.4\left(\lambda>\lambda_{1}\left(\bar{a}_{\Omega}\right)\right)$}

If $\lambda>\lambda_{1}\left(\bar{a}_{\Omega}\right)$, then

$$
A_{\mu, \lambda}\left(\phi_{1}\right)=\int_{\mathbb{R}^{N}}\left(\left|\nabla \phi_{1}\right|^{2}-\lambda a \phi_{1}^{2}\right) d x=\left(\lambda_{1}\left(\bar{a}_{\Omega}\right)-\lambda\right) \int_{\mathbb{R}^{N}} a \phi_{1}^{2} d x<0 \text { for all } \mu>0 .
$$

Hence, if $B\left(\phi_{1}\right)<0$, then by Lemma 2.1, $\mathbf{N}_{\mu, \lambda}^{+} \neq \emptyset$. Thus, as well shall see, $\mathbf{N}_{\mu, \lambda}$ may consist of two distinct components in this case which makes it possible to prove the existence of at least two positive solutions by showing that $J_{\mu, \lambda}$ has an appropriate minimizer on each component.

If $\lambda$ is just greater than $\lambda_{1}\left(\bar{a}_{\Omega}\right)$, then roughly speaking $\|u\|_{\mu}^{2} \leq \lambda \int_{\mathbb{R}^{N}} a u^{2} d x$ if and only if $u$ is almost a multiple of $\phi_{1}$ for $\mu$ enough large. Thus, if $B\left(\phi_{1}\right)<0$, it should follow that does not there exists $u \in X \backslash\{0\}$ such that $A_{\mu, \lambda}\left(\phi_{1}\right) \leq 0$ and $B\left(\phi_{1}\right) \geq 0$. This is made precise in the following lemma and we show subsequently that $\mathbf{N}_{\mu, \lambda}^{0}=\emptyset$ (or $\mathbf{N}_{\mu, \lambda}=\mathbf{N}_{\mu, \lambda}^{+} \cup \mathbf{N}_{\mu, \lambda}^{-}$) is an important condition for establishing the existence of minimizers.

Let

$$
\mathcal{A}_{\mu, \lambda}=\left\{u \in X \backslash\{0\}: A_{\mu, \lambda}(u) \leq 0\right\}
$$

and

$$
\mathcal{B}_{\mu, \lambda}=\{u \in X \backslash\{0\}: B(u) \geq 0\} .
$$

Then we have

Lemma 4.1. Suppose that $B\left(\phi_{1}\right)<0$. Then there exist $\delta_{0}>0$ and $\widehat{\mu}_{0} \geq \mu_{0}$ such that $\mathcal{A}_{\mu, \lambda} \cap \mathcal{B}_{\mu, \lambda}=\emptyset$ for all $\lambda_{1}\left(\bar{a}_{\Omega}\right)<\lambda<\lambda_{1}\left(\bar{a}_{\Omega}\right)+\delta_{0}$ and $\mu>\widehat{\mu}_{0}$. In particular, $\mathbf{N}_{\mu, \lambda}^{0}=\emptyset$ for all $\lambda_{1}\left(\bar{a}_{\Omega}\right)<\lambda<\lambda_{1}\left(\bar{a}_{\Omega}\right)+\delta_{0}$ and $\mu>\widehat{\mu}_{0}$.

Proof. Suppose that the result is false. Then there exist sequences $\left\{\mu_{n}\right\},\left\{\lambda_{n}\right\}$ and $\left\{w_{n}\right\} \subset X \backslash\{0\}$ with $\lambda_{n} \rightarrow$ $\lambda_{1}^{+}\left(\bar{a}_{\Omega}\right)$ and $\mu_{n} \rightarrow \infty$ such that

$$
A_{\mu_{n}, \lambda_{n}}\left(w_{n}\right)=\left\|w_{n}\right\|_{\mu_{n}}^{2}-\lambda_{n} \int_{\mathbb{R}^{N}} a w_{n}^{2} d x \leq 0
$$

and

$$
B\left(w_{n}\right)=\left(-\int_{\mathbb{R}^{N}}\left(I_{\alpha} \star\left|w_{n}\right|^{p}\right)\left|w_{n}\right|^{p} d x+\int_{\mathbb{R}^{N}} f\left|w_{n}\right|^{2 p} d x\right) \geq 0 .
$$

Let $u_{n}=\frac{w_{n}}{\left\|w_{n}\right\|_{\mu_{n}}}$. Since $\left\|u_{n}\right\| \leq\left\|u_{n}\right\|_{\mu_{n}}=1$, by Lemma 2.4, we may assume that there exists $u_{0} \in H_{0}^{1}(\Omega)$ such that $u_{n} \rightarrow u_{0}$ a.e. in $\mathbb{R}^{N}, u_{n} \rightarrow u_{0}$ in $L^{r}\left(\mathbb{R}^{N}\right)$ for all $2 \leq r<2^{\star}$ and $B\left(u_{n}\right) \rightarrow B\left(u_{0}\right)$. Then

$$
\lim _{n \rightarrow \infty} \lambda_{n} \int_{\mathbb{R}^{N}} a u_{n}^{2} d x=\lambda_{1}^{+}\left(\bar{a}_{\Omega}\right) \int_{\mathbb{R}^{N}} a u_{0}^{2} d x \geq 1
$$


Now, we show that $\lim _{n \rightarrow \infty} \int_{\Omega}\left|\nabla u_{n}\right|^{2} d x=\int_{\Omega}\left|\nabla u_{0}\right|^{2} d x$. Suppose on the contrary. Then by (4.1),

$$
\begin{aligned}
\int_{\Omega}\left(\left|\nabla u_{0}\right|^{2}-\lambda_{1}\left(\bar{a}_{\Omega}\right) \bar{a}_{\Omega} u_{0}^{2}\right) d x & =\int_{\mathbb{R}^{N}}\left(\left|\nabla u_{0}\right|^{2}-\lambda_{1}\left(\bar{a}_{\Omega}\right) a u_{0}^{2}\right) d x \\
& <\liminf _{n \rightarrow \infty}\left(\left\|u_{n}\right\|_{\mu_{n}}^{2}-\lambda_{n} \int_{\mathbb{R}^{N}} a u_{n}^{2} d x\right) \leq 0,
\end{aligned}
$$

which is impossible. Hence $\lim _{n \rightarrow \infty} \int_{\Omega}\left|\nabla u_{n}\right|^{2} d x=\int_{\Omega}\left|\nabla u_{0}\right|^{2} d x$. It follows that

$$
\text { (I) } \int_{\Omega}\left(\left|\nabla u_{0}\right|^{2}-\lambda_{1}\left(\bar{a}_{\Omega}\right) \bar{a}_{\Omega} u_{0}^{2}\right) d x \leq 0, \quad \text { (II) } B\left(u_{0}\right) \geq 0 .
$$

But (I) implies that $u_{0}=k \phi_{1}$ for some $k$ and then (II) implies that $k=0$ which is impossible as $\lambda_{1}^{+}\left(\bar{a}_{\Omega}\right) \int_{\mathbb{R}^{N}} a u_{0}^{2} d x \geq 1$. Thus, there exists $\delta_{0}>0$ and $\widehat{\mu}_{0} \geq \mu_{0}$ such that $\mathcal{A}_{\mu, \lambda} \cap \mathcal{B}_{\mu, \lambda}=\emptyset$ for all $\lambda_{1}\left(\bar{a}_{\Omega}\right)<$ $\lambda<\lambda_{1}\left(\bar{a}_{\Omega}\right)+\delta_{0}$ and $\mu>\widehat{\mu}_{0}$. Moreover, if $\mathbf{N}_{\mu, \lambda}^{0} \neq \emptyset$, then there exists $u_{0} \in \mathbf{N}_{\mu, \lambda}^{0}$ such that $u_{0} \in \mathcal{A}_{\mu, \lambda} \cap \mathcal{B}_{\mu, \lambda}$ which is impossible. Therefore, $\mathbf{N}_{\mu, \lambda}^{0}=\emptyset$ for all $\lambda_{1}\left(\bar{a}_{\Omega}\right)<\lambda<\lambda_{1}\left(\bar{a}_{\Omega}\right)+\delta_{0}$ and $\mu>\widehat{\mu}_{0}$. This completes the proof.

When $\mathbf{N}_{\mu, \lambda}^{0}=\emptyset$, any non-zero minimizer for $J_{\mu, \lambda}$ on $\mathbf{N}_{\mu, \lambda}^{+}$(or on $\mathbf{N}_{\mu, \lambda}^{-}$) is also a local minimizer on $\mathbf{N}_{\mu, \lambda}$ and so will be a critical point for $J_{\mu, \lambda}$ on $\mathbf{N}_{\mu, \lambda}$ and a solution of Eq. $\left(P_{\mu, \lambda}\right)$. We next show that, if $\mathbf{N}_{\mu, \lambda}^{0}=\emptyset$, it is possible to obtain more information about the nature of the Nehari manifold. Since $B\left(\phi_{1}\right)<0$, we can obtain that $\mathbf{N}_{\mu, \lambda}^{+} \neq \emptyset$ for all $\mu>0$. Furthermore, we have the following results.

Lemma 4.2. Suppose that $B\left(\phi_{1}\right)<0$. Then for any $\lambda_{1}\left(\bar{a}_{\Omega}\right)<\lambda<\lambda_{1}\left(\bar{a}_{\Omega}\right)+\delta_{0}$ and for $\mu$ enough large, we have the following results.

(i) $\mathbf{N}_{\mu, \lambda}^{+}$is uniform bounded.

(ii) There exist two negative numbers $\kappa_{1}$ and $\kappa_{2}$ such that

$$
\kappa_{1} \leq \inf _{u \in \mathbf{N}_{\mu, \lambda}^{+}} J_{\mu, \lambda}(u)<\kappa_{2} .
$$

Proof. (i) Suppose on the contrary. Then there exist sequences $\left\{\mu_{n}\right\} \subset \mathbb{R}_{+}^{N}$ and $\left\{u_{n}\right\} \subset \mathbf{N}_{\mu_{n}, \lambda}^{+}$such that $\mu_{n} \rightarrow$ $\infty$ and $\left\|u_{n}\right\|_{\mu_{n}} \rightarrow \infty$ as $n \rightarrow \infty$. Clearly,

$$
A_{\mu_{n}, \lambda}\left(u_{n}\right)=B\left(u_{n}\right)<0 .
$$

Let $v_{n}=\frac{u_{n}}{\left\|u_{n}\right\|_{\mu_{n}}}$. Then by Lemma 2.4, we may assume that there exists $v_{0} \in H_{0}^{1}(\Omega)$ such that

$$
v_{n} \rightarrow v_{0} \text { in } X ; v_{n} \rightarrow v_{0} \text { in } L^{r}\left(\mathbb{R}^{N}\right) \text { for all } 2 \leq r<2^{\star},
$$

and

$$
\lim _{n \rightarrow \infty} B\left(v_{n}\right)=B\left(v_{0}\right) .
$$

Thus,

$$
\lim _{n \rightarrow \infty} \int_{\mathbb{R}^{N}} a v_{n}^{2} d x=\int_{\mathbb{R}^{N}} a v_{0}^{2} d x
$$

Moreover, by Fatou's Lemma,

$$
\int_{\mathbb{R}^{N}}\left|\nabla v_{0}\right|^{2} d x \leq \liminf _{n \rightarrow \infty} \int_{\mathbb{R}^{N}}\left|\nabla v_{n}\right|^{2} d x
$$

Dividing (4.2) by $\left\|u_{n}\right\|_{\mu_{n}}^{2}$ gives

$$
A_{\mu_{n}, \lambda}\left(v_{n}\right)=\left\|u_{n}\right\|_{\mu_{n}}^{p-2} B\left(v_{n}\right)<0
$$


Since

$$
\lim _{n \rightarrow \infty} A_{\mu_{n}, \lambda}\left(v_{n}\right)=1-\lambda \lim _{n \rightarrow \infty} \int_{\mathbb{R}^{N}} a v_{n}^{2} d x=1-\lambda \int_{\mathbb{R}^{N}} a v_{0}^{2} d x
$$

and $\left\|u_{n}\right\|_{\mu_{n}} \rightarrow \infty$, it obtain that $B\left(v_{0}\right)=0$ and $\int_{\mathbb{R}^{N}} a v_{0}^{2} d x>0$ from the conclusions (4.3) and (4.6). Thus, $v_{0} \in \mathcal{B}_{\mu, \lambda}$ for all $\mu>0$. Moreover, by $v_{0} \in H_{0}^{1}(\Omega)$, (4.5) and (4.4), for every $\mu>0$,

$$
\left\|v_{0}\right\|_{\mu}^{2}-\lambda \int_{\mathbb{R}^{N}} a v_{0}^{2} d x=\int_{\mathbb{R}^{N}}\left|\nabla v_{0}\right|^{2}-\lambda a v_{0}^{2} d x<\liminf _{n \rightarrow \infty} A_{\mu_{n}, \lambda}\left(v_{n}\right) \leq 0,
$$

which indicates that $v_{0} \in \mathcal{A}_{\mu, \lambda}$. We now show that $v_{n} \rightarrow v_{0}$ in $X_{\mu}$. Suppose on the contrary. Then

$$
\lambda\left\|v_{0}\right\|_{\mu}^{2}-\int_{\mathbb{R}^{N}} a v_{0}^{2} d x=\int_{\mathbb{R}^{N}}\left|\nabla v_{0}\right|^{2}-\lambda a v_{0}^{2} d x<\lim _{n \rightarrow \infty} A_{\mu_{n}, \lambda}\left(v_{n}\right) \leq 0,
$$

since $\int_{\mathbb{R}^{N}} g v_{0}^{2} d x=0$. Hence $v_{0} \in \mathcal{A}_{\mu, \lambda} \cap \mathcal{B}_{\mu, \lambda}$ which is impossible. Since $v_{n} \rightarrow v_{0}$ in $X_{\mu}$, then $\left\|v_{0}\right\|_{\mu}=1$. Hence $v_{0} \in \mathcal{B}_{\mu, \lambda}$. Moreover,

$$
\left\|v_{0}\right\|_{\mu}^{2}-\lambda \int_{\mathbb{R}^{N}} a v_{0}^{2} d x=\lim _{n \rightarrow \infty} A_{\mu_{n}, \lambda}\left(v_{n}\right) \leq 0
$$

and so $v_{0} \in \mathcal{A}_{\mu, \lambda}$. Thus, $v_{0} \in \mathcal{A}_{\mu, \lambda} \cap \mathcal{B}_{\mu, \lambda}$ which is impossible. Hence $\mathbf{N}_{\mu, \lambda}^{+}$is uniform bounded for $\mu>0$ sufficiently large.

(ii) By part (i), there exists $C_{0}>0$ such that $\|u\|_{\mu} \leq C_{0}$ for all $u \in \mathbf{N}_{\mu, \lambda}^{+}$. Hence, making use of (2.1), for $u \in \mathbf{N}_{\mu, \lambda}^{+}$ we have

$$
\begin{aligned}
J_{\mu, \lambda}(u) & =\frac{p-1}{2 p} B(u) \geq-\frac{p-1}{2 p}\left(\int_{\mathbb{R}^{N}}\left(I_{\alpha}{ }^{\star}|u|^{p}\right)|u|^{p} d x+\|f\|_{\infty} \int_{\mathbb{R}^{N}}|u|^{2 p} d x\right) \\
& \geq-\frac{p-1}{2 p} C_{1}\|u\|_{\mu}^{2 p} \geq-\frac{p-1}{2 p S^{p}} C_{1} C_{0}^{p}=\kappa_{1} .
\end{aligned}
$$

Moreover, by $B\left(\phi_{1}\right)<0$ and $\int_{\Omega}\left|\nabla \phi_{1}\right|^{2} d x-\lambda \int_{\Omega} a \phi_{1}^{2} d x<0$, which indicates that the function $h_{\phi_{1}}(t)=$ $J_{\mu, \lambda}\left(t \phi_{1}\right)$ have $t_{0}^{+}>0$ and $\kappa_{2}<0$ are independent of $\mu$ such that $t_{0}^{+} \varphi \in \mathbf{N}_{\mu, \lambda}^{+}$and

$$
\inf _{0<t<\infty} h_{\phi_{1}}(t)=h_{\phi_{1}}\left(t_{0}^{+}\right)=\kappa_{2}<0 \text {. }
$$

This implies that

$$
\inf _{u \in \mathbf{N}_{\mu, \lambda}^{+}} J_{\mu, \lambda}(u) \leq \kappa_{2}<0 \text { for all } \mu>\max \left\{\bar{\mu}_{1}, \bar{\mu}_{2}\right\}
$$

This completes the proof.

Theorem 4.3. Suppose that $B\left(\phi_{1}\right)<0$. Then for any $\lambda_{1}\left(\bar{a}_{\Omega}\right)<\lambda<\lambda_{1}\left(\bar{a}_{\Omega}\right)+\delta_{0}$ and for $\mu$ enough large, there exists a minimizer of $J_{\mu, \lambda}(u)$ on $\mathbf{N}_{\mu, \lambda}^{+}$.

Proof. By Lemmas 4.1, 4.2 and the Ekeland variational principle [19], there exists a minimizing sequence $\left\{u_{n}\right\} \subset \mathbf{N}_{\mu, \lambda}^{+}$such that

$$
\lim _{n \rightarrow \infty} J_{\mu, \lambda}\left(u_{n}\right)=\inf _{u \in \mathbf{N}_{\mu, \lambda}^{+}} J_{\mu, \lambda}(u) \leq \kappa_{2} \text { and } J_{\mu, \lambda}^{\prime}\left(u_{n}\right)=o(1)
$$

and there exists $\bar{C}_{0}>0$ such that $\left\|u_{n}\right\|_{\mu} \leq \bar{C}_{0}$. Thus, there exist a subsequence $\left\{u_{n}\right\}$ and $u_{0} \in X_{\mu}$ such that $J_{\mu, \lambda}^{\prime}\left(u_{0}\right)=0$ and

$$
\begin{aligned}
& u_{n} \rightarrow u_{0} \text { in } X_{\mu}, \\
& u_{n} \rightarrow u_{0} \text { a.e. in } \mathbb{R}^{N}, \\
& u_{n} \rightarrow u_{0} \text { in } L_{l o c}^{r}\left(\mathbb{R}^{N}\right) \text { for } 2 \leq r<2^{\star} .
\end{aligned}
$$


Then by condition $\left(V_{4}\right)$,

$$
\lim _{n \rightarrow \infty} \int_{\mathbb{R}^{N}} a u_{n}^{2} d x=\int_{\mathbb{R}^{N}} a u_{0}^{2} d x
$$

Moreover, follows from Brezis-Lieb lemma [8] and Lemma 2.3, obtain that

$$
B\left(u_{n}-u_{0}\right)=B\left(u_{n}\right)-B\left(u_{0}\right)+o(1) .
$$

Now we prove that $u_{n} \rightarrow u_{0}$ in $X_{\mu}$. Let $v_{n}=u_{n}-u_{0}$. Then $v_{n} \rightarrow 0$ in $X_{\mu}$. By the Sobolev and GagliardoNirenberg inequalities, for any $\mu>\bar{\mu}_{0}$ we have that

$$
\int_{\mathbb{R}^{N}} v_{n}^{2} d x \leq \frac{1}{\mu c} \int_{\{g \geq c\}} \mu g v_{n}^{2} d x+\int_{\{g<c\}} v_{n}^{2} d x \leq \frac{1}{\mu c} \int_{\mathbb{R}^{N}} \mu g v_{n}^{2} d x+o(1)
$$

and

$$
\begin{aligned}
\int_{\mathbb{R}^{N}}\left|v_{n}\right|^{2 p} d x & \leq C_{0}\left(\frac{1}{\mu c}\left\|v_{n}\right\|_{\mu}^{2}\right)^{\frac{2^{*}-2 p}{2^{*}-2}}\left(\int_{\mathbb{R}^{N}}\left|\nabla v_{n}\right|^{2} d x\right)^{\frac{2^{*}(p-1)}{2^{*}-2}}+o(1) \\
& \leq C_{0}\left(\frac{1}{\mu c}\right)^{\frac{2^{*}-2 p}{2^{*}-2}}\left\|v_{n}\right\|_{\mu}^{2 p}+o(1)
\end{aligned}
$$

or

$$
\int_{\mathbb{R}^{N}}\left|v_{n}\right|^{2 p} d x \leq \Pi_{\mu}\left\|v_{n}\right\|_{\mu}^{2 p}+o(1)
$$

where $\Pi_{\mu}=C_{0}\left(\frac{1}{\mu c}\right)^{\frac{2^{*}-2 p}{2^{*}-2}}$. Thus, using (4.9) and (4.10) gives

$$
J_{\mu, \lambda}\left(v_{n}\right)=J_{\mu, \lambda}\left(u_{n}\right)-J_{\mu, \lambda}\left(u_{0}\right)+o(1) \text { and }\left\langle J_{\mu, \lambda}^{\prime}\left(v_{n}\right), v_{n}\right\rangle=o(1) .
$$

Consequently, by (4.7) , (4.9), (4.12) and Lemma 4.2 (ii), one has

$$
\begin{aligned}
\kappa_{2}+\left|\kappa_{1}\right| & \geq \inf _{u \in \mathbf{N}_{\mu, \lambda}^{+}} J_{\mu, \lambda}(u)-J_{\mu, \lambda}\left(u_{0}\right) \geq J_{\mu, \lambda}\left(v_{n}\right)-\frac{1}{2 p}\left\langle J_{\mu, \lambda}^{\prime}\left(v_{n}\right), v_{n}\right\rangle+o(1) \\
& \geq \frac{p-1}{2 p}\left\|v_{n}\right\|_{\mu}^{2}+o(1),
\end{aligned}
$$

which shows that there exists a constant $C_{1}>0$ such that

$$
\left\|v_{n}\right\|_{\mu} \leq C_{1}+o(1) \text { for } \mu>0 \text { sufficiently large. }
$$

Since $1<\frac{N+\alpha}{N}<p<2_{\alpha}^{\star}$, it follows from (4.9), (4.11) and (4.13) that

$$
\begin{aligned}
o(1) & =\left\langle J_{\lambda}^{\prime}\left(v_{n}\right), v_{n}\right\rangle \geq\left\|v_{n}\right\|_{\mu}^{2}\left(1-C_{0} \Pi_{\mu}\left\|v_{n}\right\|_{\mu}^{2 p-2}\right)+o(1) \\
& \geq\left\|v_{n}\right\|_{\mu}^{2}\left(1-\|f\|_{\infty} \Pi_{\mu} C_{1}^{2 p-2}\right)+o(1) .
\end{aligned}
$$

Notice that $\Pi_{\mu} \rightarrow 0$ as $\mu \rightarrow \infty$. Then by (4.14), there holds $v_{n} \rightarrow 0$ in $X_{\mu}$ for $\mu>0$ sufficiently large. Hence $u_{n} \rightarrow u_{0}$ in $X_{\mu}$ and so

$$
J_{\mu, \lambda}\left(u_{0}\right)=\lim _{n \rightarrow \infty} J_{\mu, \lambda}\left(u_{n}\right)=\inf _{u \in \mathbf{N}_{\mu, \lambda}^{+}} J_{\mu, \lambda}(u) \leq \kappa_{0}<0,
$$

which implies that $u_{0}$ is a minimizer on $\mathbf{N}_{\mu, \lambda}^{+}$.

We now turn our attention to $\mathbf{N}_{\mu, \lambda}^{-}$. 
Lemma 4.4. Suppose that $B\left(\phi_{1}\right)<0$. Then for any $\lambda_{1}\left(\bar{a}_{\Omega}\right)<\lambda<\lambda_{1}\left(\bar{a}_{\Omega}\right)+\delta_{0}$ and for $\mu$ enough large, we have the following results

(i) there exists $c_{0}>0$ such that $\|u\|_{\mu} \geq c_{0}$ for all $u \in \mathbf{N}_{\mu, \lambda}^{-}$;

(ii) every minimizing sequence for $J_{\mu, \lambda}(u)$ on $\mathbf{N}_{\mu, \lambda}^{-}$is bounded;

(iii) $\inf _{u \in \mathbf{N}_{\mu, \lambda}^{-}} J_{\mu, \lambda}(u)>0$.

Proof. (i) Suppose on the contrary. Then there exist $\left\{\mu_{n}\right\} \subset \mathbb{R}^{+}$and $\left\{u_{n}\right\} \subset \mathbf{N}_{\mu_{n}, \lambda}^{-}$such that $\mu_{n} \rightarrow \infty$ and $\left\|u_{n}\right\|_{\mu_{n}} \rightarrow 0$. Hence, by (2.1),

$$
0<A_{\mu_{n}, \lambda}\left(u_{n}\right)=B\left(u_{n}\right) \rightarrow 0 \text { as } n \rightarrow \infty .
$$

Let $v_{n}=\frac{u_{n}}{\left\|u_{n}\right\|_{\mu_{n}}}$. Then, by Lemma 2.4, there exist subsequence $\left\{v_{n}\right\}$ and $v_{0} \in H_{0}^{1}(\Omega)$ such that

$$
v_{n} \rightarrow v_{0} \text { in } X ; v_{n} \rightarrow v_{0} \text { in } L^{r}\left(\mathbb{R}^{N}\right) \text { for all } 2 \leq r<2^{\star} .
$$

Thus,

$$
\lim _{n \rightarrow \infty} \int_{\mathbb{R}^{N}} a v_{n}^{2} d x=\int_{\mathbb{R}^{N}} a v_{0}^{2} d x
$$

and

$$
A_{\mu_{n}, \lambda}\left(v_{n}\right)=\left\|u_{n}\right\|_{\mu_{n}}^{2 p-2} B\left(v_{n}\right) \rightarrow 0 \text { as } n \rightarrow \infty .
$$

Moreover, by (4.15), (4.16), $v_{0} \in H_{0}^{1}(\Omega)$ and Fatou's Lemma, we can obtain that

$$
0=\lim _{n \rightarrow \infty} A_{\mu_{n}, \lambda}\left(v_{n}\right)=1-\lambda \lim _{n \rightarrow \infty} \int_{\mathbb{R}^{N}} a v_{n}^{2} d x=1-\lambda \int_{\mathbb{R}^{N}} a v_{0}^{2} d x,
$$

and for every $\mu>0$

$$
\begin{aligned}
\left\|v_{0}\right\|_{\mu}^{2}-\int_{\mathbb{R}^{N}} \lambda a v_{0}^{2} d x & =\int_{\mathbb{R}^{N}}\left|\nabla v_{0}\right|^{2}-\lambda a v_{0}^{2} d x \\
& \leq \liminf _{n \rightarrow \infty}\left(\left\|v_{n}\right\|_{\mu_{n}}^{2}-\int_{\mathbb{R}^{N}} \lambda a v_{n}^{2} d x\right)=0,
\end{aligned}
$$

this implies that $v_{0} \neq 0$ and $v_{0} \in \mathcal{A}_{\mu, \lambda}$ for all $\mu>0$. Since $B\left(v_{n}\right)>0$ and $B\left(v_{n}\right) \rightarrow B\left(v_{0}\right)$, it follows that $\frac{v_{0}}{\left\|v_{0}\right\|_{\mu}} \in \mathcal{B}_{\mu, \lambda}$ for all $\mu>0$. Hence, $v_{0} \in \mathcal{A}_{\mu, \lambda} \cap \mathcal{B}_{\mu, \lambda}$ for all $\mu>0$, which a contradiction.

(ii) Suppose on the contrary. Then there exist sequences $\left\{\mu_{n}\right\} \subset \mathbb{R}^{+}$with $\mu_{n} \rightarrow \infty$ such that $\mathbf{N}_{\mu_{n}, \lambda}^{-}$is unbounded for all $n$, that is for every $n$ there exists a minimizing sequence $\left\{u_{n, m}\right\} \subset \mathbf{N}_{\mu_{n}, \lambda}^{-}$such that $\left\|u_{n, m}\right\|_{\mu_{n}} \rightarrow \infty$ as $m \rightarrow \infty$. Moreover,

$$
A_{\mu_{n}, \lambda}\left(u_{n, m}\right)=B\left(u_{n, m}\right) \rightarrow \frac{p-1}{2 p} \inf _{u \in \mathbf{N}_{\mu_{n}, \lambda}^{-}} J_{\mu_{n}, \lambda}(u) \text { as } m \rightarrow \infty,
$$

where $\inf _{u \in \mathbf{N}_{\mu_{n}, \lambda}^{-}} J_{\mu_{n}, \lambda}(u) \geq 0$ for all $n$. Let $w_{n}=u_{n, n}$. Then $w_{n} \in \mathbf{N}_{\mu_{n}, \lambda}^{-}$and $\left\|w_{n}\right\|_{\mu_{n}} \rightarrow \infty$ as $n \rightarrow \infty$. Let $v_{n}=\frac{w_{n}}{\left\|w_{n}\right\|_{\mu_{n}}}$. Then by Lemma 2.4, we may assume that there exist subsequence $\left\{v_{n}\right\}$ and $v_{0} \in H_{0}^{1}(\Omega)$ such that $v_{n} \rightarrow v_{0}$ in $X, v_{n} \rightarrow v_{0}$ in $L^{r}\left(\mathbb{R}^{N}\right)$ for all $2 \leq r<2^{*}$ and $B\left(v_{n}\right) \rightarrow B\left(v_{0}\right)$. Then by condition $\left(V_{4}\right)$

$$
\lim _{n \rightarrow \infty} \int_{\mathbb{R}^{N}} a v_{n}^{2} d x=\int_{\mathbb{R}^{N}} a v_{0}^{2} d x
$$

Dividing (4.17) by $\left\|w_{n}\right\|_{\mu_{n}}^{2}$ and $m=n$ gives

$$
A_{\mu_{n}, \lambda}\left(v_{n}\right)=\left\|w_{n}\right\|_{\mu_{n}}^{p-2} B\left(v_{n}\right) \rightarrow 0 .
$$


Since $\left\|w_{n}\right\| \mu_{n} \rightarrow+\infty$, it follows that $B\left(v_{n}\right) \rightarrow 0$ and so $B\left(v_{0}\right)=0$. We now show that $v_{n} \rightarrow v_{0}$ in $X$. Suppose otherwise, then by (4.18) and (4.19),

$$
\begin{aligned}
\int_{\mathbb{R}^{N}}\left|\nabla v_{0}\right|^{2}-\lambda a v_{0}^{2} d x & =\left\|v_{0}\right\|^{2}-\lambda \int_{\mathbb{R}^{N}} a v_{0}^{2} d x \\
& <\liminf _{n \rightarrow \infty}\left(\left\|v_{n}\right\|_{\mu_{n}}^{2}-\lambda \int_{\mathbb{R}^{N}} a v_{n}^{2} d x\right)=0 .
\end{aligned}
$$

Thus, $v_{0} \neq 0$ and for every $\mu>0$, there holds $v_{0} \in \mathcal{A}_{\mu, \lambda} \cap \mathcal{B}_{\mu, \lambda}$, which is impossible. Hence $v_{n} \rightarrow v_{0}$ in $X$. It follows that $\left\|v_{0}\right\|_{\mu}=1, \int_{\mathbb{R}^{N}} V v_{0}^{2} d x=0$ and

$$
\left\|v_{0}\right\|_{\mu}^{2}-\lambda \int_{\mathbb{R}^{N}} a v_{0}^{2} d x=B\left(v_{0}\right)=0
$$

Thus, for every $\mu>0$, there holds $v_{0} \in \mathcal{A}_{\mu, \lambda} \cap \mathcal{B}_{\mu, \lambda}$ which is impossible as $\mathcal{A}_{\mu, \lambda} \cap \mathcal{B}_{\mu, \lambda}=\emptyset$. Hence, every minimizing sequence for $J_{\mu, \lambda}(u)$ on $\mathbf{N}_{\mu, \lambda}^{-}$is bounded for $\mu$ sufficiently large.

(iii) Assume that $\inf _{u \in \mathbf{N}_{\mu, \lambda}^{-}} J_{\mu, \lambda}(u)=0$. Then by the Ekeland variational principle [19], there exists a minimizing sequence $\left\{u_{n}\right\} \subset \mathbf{N}_{\mu, \lambda}^{-}$such that

$$
\lim _{n \rightarrow \infty} J_{\mu, \lambda}\left(u_{n}\right)=\inf _{u \in \mathbf{N}_{\mu, \lambda}^{-}} J_{\mu, \lambda}(u) \text { and } J_{\mu, \lambda}^{\prime}\left(u_{n}\right)=o(1) .
$$

By part (ii), $\left\{u_{n}\right\}$ is bounded and so there exist a subsequence $\left\{u_{n}\right\}$ and $u_{0} \in X_{\mu}$ such that $J_{\mu, \lambda}^{\prime}\left(u_{0}\right)=0$ and

$$
\begin{aligned}
& u_{n} \rightarrow u_{0} \text { in } X_{\mu}, \\
& u_{n} \rightarrow u_{0} \text { a.e. in } \mathbb{R}^{N}, \\
& u_{n} \rightarrow u_{0} \text { in } L_{l o c}^{r}\left(\mathbb{R}^{N}\right) \text { for } 2 \leq r<2^{*} .
\end{aligned}
$$

Then by condition $\left(V_{4}\right)$

$$
\lim _{n \rightarrow \infty} \int_{\mathbb{R}^{N}} a v_{n}^{2} d x=\int_{\mathbb{R}^{N}} a v_{0}^{2} d x
$$

Moreover, follows from Brezis-Lieb lemma [8] and Lemma 2.3, obtain that

$$
B\left(u_{n}-u_{0}\right)=B\left(u_{n}\right)-B\left(u_{0}\right)+o(1) .
$$

Now we prove that $u_{n} \rightarrow u_{0}$ in $X_{\mu}$. Let $v_{n}=u_{n}-u_{0}$. Then $v_{n} \rightarrow 0$ in $X_{\mu}$. By the Sobolev and GagliardoNirenberg inequalities, for $\mu$ enough large we have that

$$
\int_{\mathbb{R}^{N}} v_{n}^{2} d x \leq \frac{1}{\mu c} \int_{\{g \geq c\}} \mu g v_{n}^{2} d x+\int_{\{g<c\}} v_{n}^{2} d x \leq \frac{1}{\mu c} \int_{\mathbb{R}^{N}} \mu g v_{n}^{2} d x+o(1)
$$

and

$$
\begin{aligned}
\int_{\mathbb{R}^{N}}\left|v_{n}\right|^{2 p} d x & \leq C_{0}\left(\frac{1}{\mu c}\left\|v_{n}\right\|_{\mu}^{2}\right)^{\frac{2^{*}-2 p}{2^{*}-2}}\left(\int_{\mathbb{R}^{N}}\left|\nabla v_{n}\right|^{2} d x\right)^{\frac{2^{*}(p-1)}{2^{*}-2}}+o(1) \\
& \leq C_{0}\left(\frac{1}{\mu c}\right)^{\frac{2^{*}-2 p}{2^{*}-2}}\left\|v_{n}\right\|_{\mu}^{2 p}+o(1) .
\end{aligned}
$$

or

$$
\int_{\mathbb{R}^{N}}\left|v_{n}\right|^{2 p} d x \leq \Pi_{\mu}\left\|v_{n}\right\|_{\mu}^{2 p}+o(1)
$$


where $\Pi_{\mu}=C_{0}\left(\frac{1}{\mu b}\right)^{\frac{2^{*}-2 p}{2^{*}-2}} S^{-N(p-1)}$. Thus, using (4.21) and $u_{n} \rightarrow u_{0}$ in $X_{\mu}$ gives

$$
J_{\mu, \lambda}\left(v_{n}\right)=J_{\mu, \lambda}\left(u_{n}\right)-J_{\mu, \lambda}\left(u_{0}\right)+o(1) \text { and }\left\langle J_{\mu, \lambda}^{\prime}\left(v_{n}\right), v_{n}\right\rangle=o(1) .
$$

Consequently, by (4.20), (4.21) and (4.23), one has

$$
\begin{aligned}
\inf _{u \in \mathbf{N}_{\mu, \lambda}^{-}} J_{\mu, \lambda}(u)-J_{\mu, \lambda}\left(u_{0}\right) & \geq J_{\mu, \lambda}\left(v_{n}\right)-\frac{1}{2 p}\left\langle J_{\mu, \lambda}^{\prime}\left(v_{n}\right), v_{n}\right\rangle+o(1) \\
& \geq \frac{p-1}{2 p}\left\|v_{n}\right\|_{\mu}^{2}+o(1) .
\end{aligned}
$$

Suppose that $\inf _{u \in \mathbf{N}_{\mu, \lambda}^{-}} J_{\mu, \lambda}(u)=0$.

(iii - A) If $u_{0} \in \mathbf{N}_{\mu, \lambda}^{-}$, then by (4.24) and $u_{0}=0,\left\|v_{n}\right\|_{\mu}^{2} \rightarrow 0$, this shows that $u_{n} \rightarrow u_{0}$ in $X_{\mu}$, and so

$$
J_{\mu, \lambda}\left(u_{0}\right)=\lim _{n \rightarrow \infty} J_{\mu, \lambda}\left(u_{n}\right)=\inf _{u \in \mathbf{N}_{\mu, \lambda}^{-}} J_{\mu, \lambda}(u)=0 .
$$

It then follows exactly as in the proof in part (i) that $u_{0} \in \mathcal{A}_{\mu, \lambda} \cap \mathcal{B}_{\mu, \lambda}$ which is impossible as $\mathcal{A}_{\mu, \lambda} \cap \mathcal{B}_{\mu, \lambda}=\emptyset$. (iii - B) If $u_{0} \in \mathbf{N}_{\mu, \lambda}^{+}$, then by (4.7) and (4.24), there exists $C_{0}>0$ such that

$$
\left\|v_{n}\right\|_{\mu} \leq C_{0}+o(1) \text { for } \mu \text { enough large. }
$$

Since $1<\frac{N+\alpha}{N}<p<2_{\alpha}^{\star}$, it follows from (4.18), (4.22) and (4.25) that

$$
\begin{aligned}
o(1) & =\left\langle J_{\mu, \lambda}^{\prime}\left(v_{n}\right), v_{n}\right\rangle \geq\left\|v_{n}\right\|_{\mu}^{2}\left(1-\|f\|_{\infty} \Pi_{\mu}\left\|v_{n}\right\|_{\mu}^{2 p-2}\right)+o(1) \\
& \geq\left\|v_{n}\right\|_{\mu}^{2}\left(1-\|f\|_{\infty} \Pi_{\mu} C_{0}^{2 p-2}\right)+o(1) .
\end{aligned}
$$

Notice that $\Pi_{\mu} \rightarrow 0$ as $\mu \rightarrow \infty$. Then by (4.26), for $\mu$ enough large, there holds $v_{n} \rightarrow 0$ in $X_{\mu}$. Hence $u_{n} \rightarrow u_{0}$ in $X_{\mu}$, and so $u_{0} \in \mathbf{N}_{\mu, \lambda}^{-}$this is a contradiction. Thus, $\inf _{u \in \mathbf{N}_{\mu, \lambda}^{-}} J_{\mu, \lambda}(u)>0$ for $\mu$ enough large. This completes the proof.

Theorem 4.5. Suppose that $B\left(\phi_{1}\right)<0$. Then for any $\lambda_{1}\left(\bar{a}_{\Omega}\right)<\lambda<\lambda_{1}\left(\bar{a}_{\Omega}\right)+\delta_{0}$ and for $\mu$ enough large, there exists a minimizer of $J_{\mu, \lambda}(u)$ on $\mathbf{N}_{\mu, \lambda}^{-}$.

Proof. By Lemmas 4.1, 4.4 (iii) and the Ekeland variational principle [19], there exists a minimizing sequence $\left\{u_{n}\right\} \subset \mathbf{N}_{\mu, \lambda}^{-}$such that

$$
\lim _{n \rightarrow \infty} J_{\mu, \lambda}\left(u_{n}\right)=\inf _{u \in \mathbf{N}_{\mu, \lambda}^{-}} J_{\mu, \lambda}(u) \text { and } J_{\mu, \lambda}^{\prime}\left(u_{n}\right)=o(1) .
$$

Similar the argument in (3.3), there exists $D_{0}>0$ independent of $\mu$ such that $\inf _{u \in \mathbf{N}_{\mu, \lambda}^{-}} J_{\mu, \lambda}(u)<D_{0}$ for all $\mu \geq \mu_{0}$. Moreover, by Lemma 4.4 (ii), there exists $C_{0}>0$ such that $\left\|u_{n}\right\|_{\mu} \leq C_{0}$. Thus, there exist a subsequence $\left\{u_{n}\right\}$ and $u_{0} \in X_{\mu}$ such that $J_{\mu, \lambda}^{\prime}\left(u_{0}\right)=0$ and

$$
\begin{aligned}
& u_{n} \rightarrow u_{0} \text { in } X_{\mu}, \\
& u_{n} \rightarrow u_{0} \text { a.e. in } \mathbb{R}^{N}, \\
& u_{n} \rightarrow u_{0} \text { in } L_{l o c}^{r}\left(\mathbb{R}^{N}\right) \text { for } 2 \leq r<2^{*} .
\end{aligned}
$$

Then by condition $\left(V_{4}\right)$,

$$
\lim _{n \rightarrow \infty} \int_{\mathbb{R}^{N}} a v_{n}^{2} d x=\int_{\mathbb{R}^{N}} a v_{0}^{2} d x,
$$

and follows from Brezis-Lieb lemma [8] and Lemma 2.3 obtain that

$$
B\left(u_{n}-u_{0}\right)=B\left(u_{n}\right)-B\left(u_{0}\right)+o(1) .
$$


Now we prove that $u_{n} \rightarrow u_{0}$ in $X_{\mu}$. Let $v_{n}=u_{n}-u_{0}$. Then $v_{n} \rightarrow 0$ in $X_{\mu}$. By the Sobolev and GagliardoNirenberg inequalities, for $\mu$ enough large we have that

$$
\int_{\mathbb{R}^{N}} v_{n}^{2} d x \leq \frac{1}{\mu c} \int_{\{g \geq c\}} \mu g v_{n}^{2} d x+\int_{\{g<c\}} v_{n}^{2} d x \leq \frac{1}{\mu c} \int_{\mathbb{R}^{N}} \mu g v_{n}^{2} d x+o(1)
$$

and

$$
\begin{aligned}
\int_{\mathbb{R}^{N}}\left|v_{n}\right|^{2 p} d x & \leq C_{0}\left(\frac{1}{\mu c}\left\|v_{n}\right\|_{\mu}^{2}\right)^{\frac{2^{*}-2 p}{2^{*}-2}}\left(\int_{\mathbb{R}^{N}}\left|\nabla v_{n}\right|^{2} d x\right)^{\frac{2^{*}(p-1)}{2^{*}-2}}+o(1) \\
& \leq C_{0}\left(\frac{1}{\mu c}\right)^{\frac{2^{*}-2 p}{2^{*}-2}}\left\|v_{n}\right\|_{\mu}^{2 p}+o(1)
\end{aligned}
$$

or

$$
\int_{\mathbb{R}^{N}}\left|v_{n}\right|^{2 p} d x \leq \Pi_{\mu}\left\|v_{n}\right\|_{\mu}^{2 p}+o(1),
$$

where $\Pi_{\mu}=C_{0}\left(\frac{1}{\mu c}\right)^{\frac{2^{*}-2 p}{2^{*}-2}}$. Thus, using (4.27) and (4.28) gives

$$
J_{\mu, \lambda}\left(v_{n}\right)=J_{\mu, \lambda}\left(u_{n}\right)-J_{\mu, \lambda}\left(u_{0}\right)+o(1) \text { and } J_{\mu, \lambda}^{\prime}\left(v_{n}\right)=o(1) .
$$

Consequently, by (4.27), (4.30) and Lemma 4.2 (ii), one has

$$
\begin{aligned}
D_{0}+\left|\kappa_{1}\right| & \geq \inf _{u \in \mathbf{N}_{\mu, \lambda}^{-}} J_{\mu, \lambda}(u)-J_{\mu, \lambda}\left(u_{0}\right) \geq J_{\mu, \lambda}\left(v_{n}\right)-\frac{1}{p}\left\langle J_{\mu, \lambda}^{\prime}\left(v_{n}\right), v_{n}\right\rangle+o(1) \\
& \geq \frac{p-2}{2 p}\left\|v_{n}\right\|_{\mu}^{2}+o(1),
\end{aligned}
$$

which shows that there exists a constant $C_{1}>0$ such that for $\mu$ enough large,

$$
\left\|v_{n}\right\|_{\mu} \leq C_{1}+o(1) \text {. }
$$

Since $1<\frac{N+\alpha}{N}<p<2_{\alpha}^{\star}$, it follows from (4.29) (4.31) and (4.32) that

$$
\begin{aligned}
o(1) & =\left\langle J_{\lambda}^{\prime}\left(v_{n}\right), v_{n}\right\rangle \geq\left\|v_{n}\right\|_{\mu}^{2}\left(1-\|f\|_{\infty} \Pi_{\mu}\left\|v_{n}\right\|_{\mu}^{2 p-2}\right)+o(1) \\
& \geq\left\|v_{n}\right\|_{\mu}^{2}\left(1-\|f\|_{\infty} \Pi_{\mu} C_{1}^{2 p-2}\right)+o(1) .
\end{aligned}
$$

Notice that $\Pi_{\mu} \rightarrow 0$ as $\mu \rightarrow \infty$. Then by (4.33), for $\mu$ enough large, there holds $v_{n} \rightarrow 0$ in $X_{\mu}$. Hence $u_{n} \rightarrow u_{0}$ in $X_{\mu}$ and so

$$
J_{\mu, \lambda}\left(u_{0}\right)=\lim _{n \rightarrow \infty} J_{\mu, \lambda}\left(u_{n}\right)=\inf _{u \in \mathbf{N}_{\mu, \lambda}^{-}} J_{\mu, \lambda}(u),
$$

which implies that $u_{0}$ is a minimizer on $\mathbf{N}_{\mu, \lambda}^{-}$.

We are now ready to prove Theorem 1.3: By Theorem 4.5 and 4.3, there exist $\delta_{0}$ such that when $\lambda_{1}\left(\bar{a}_{\Omega}\right)<$ $\lambda<\lambda_{1}\left(\bar{a}_{\Omega}\right)+\delta_{0}$ and for $\mu$ enough large, $J_{\mu, \lambda}$ has minimizers in each of $\mathbf{N}_{\mu, \lambda}^{(1)}$ and $\mathbf{N}_{\mu, \lambda}^{(2)}$, that is there exist $u_{\mu, \lambda}^{(1)} \in \mathbf{N}_{\mu, \lambda}^{+}$and $u_{\mu, \lambda}^{(2)} \in \mathbf{N}_{\mu, \lambda}^{-}$such that

$$
J_{\mu, \lambda}\left(u_{\mu, \lambda}^{(1)}\right)=\inf _{u \in \mathbf{N}_{\mu, \lambda}^{+}} J_{\mu, \lambda}(u)<\kappa_{2}<0<\inf _{u \in \mathbf{N}_{\mu, \lambda}^{-}} J_{\mu, \lambda}(u)=J_{\mu, \lambda}\left(u_{\mu, \lambda}^{(2)}\right) .
$$

Since $J_{\mu, \lambda}\left(u_{\mu, \lambda}^{(j)}\right)=J_{\mu, \lambda}\left(\left|u_{\mu, \lambda}^{(j)}\right|\right)$ for $j=1,2$, we may assume that these minimizers are positive. Moreover, by Lemma 4.1, $\mathbf{N}_{\mu, \lambda}=\mathbf{N}_{\mu, \lambda}^{+} \cup \mathbf{N}_{\mu, \lambda}^{-}$. It follows that the minimizers are local minimizers in $\mathbf{N}_{\mu, \lambda}$ which do not lie in $\mathbf{N}_{\mu, \lambda}^{0}$, and so by Lemma 2.2, $u_{\mu, \lambda}^{(1)}$ and $u_{\mu, \lambda}^{(2)}$ are positive solutions of Eq. $\left(P_{\mu, \lambda}\right)$. This completes the proof. 
Next, we are ready to prove Theorem 1.4: (i) Since $\mathbf{N}_{\mu_{n}, \lambda_{n}}^{+}$is uniformly bounded, then $\left\{u_{n}\right\}$ is bounded, from Lemma 2.4, we may assume that there exists $u_{0} \in H_{0}^{1}(\Omega)$ such that $u_{n} \rightarrow u_{0}$ in $X, u_{n} \rightarrow u_{0}$ in $L^{r}\left(\mathbb{R}^{N}\right)$ for all $2 \leq r<2^{\star}$ and $B\left(u_{n}\right) \rightarrow B\left(u_{0}\right)$. We also have

$$
\lim _{n \rightarrow \infty} \int_{\mathbb{R}^{N}} a u_{n}^{2} d x=\int_{\mathbb{R}^{N}} a u_{0}^{2} d x
$$

and

$$
\left\|u_{n}\right\|^{2}-\lambda_{n} \int_{\mathbb{R}^{N}} a u_{n}^{2} d x \leq A_{\mu_{n}, \lambda_{n}}\left(u_{n}\right)=B\left(u_{n}\right)<0 \text { for } n \text { sufficiently large. }
$$

We now show that $u_{n} \rightarrow u_{0}$ in $X$. Suppose on the contrary. Then

$$
\begin{aligned}
\int_{\mathbb{R}^{N}}\left(\left|\nabla u_{0}\right|^{2}-\lambda_{1}\left(\bar{a}_{\Omega}\right) a u_{0}^{2}\right) d x & =\int_{\mathbb{R}^{N}}\left(\left|\nabla u_{0}\right|^{2}+V u_{0}^{2}-\lambda_{1}\left(\bar{a}_{\Omega}\right) a u_{0}^{2}\right) d x \\
& <\liminf _{n \rightarrow \infty}\left(\left\|u_{n}\right\|^{2}-\lambda_{n} \int_{\mathbb{R}^{N}} a u_{n}^{2} d x\right) \leq 0,
\end{aligned}
$$

which is impossible. Thus, $u_{n} \rightarrow u_{0}$ in $X$ and so

$$
\begin{aligned}
\int_{\Omega}\left(\left|\nabla u_{0}\right|^{2}-\lambda_{1}\left(\bar{a}_{\Omega}\right) a u_{0}^{2}\right) d x & \leq \int_{\mathbb{R}^{N}}\left(\left|\nabla u_{0}\right|^{2}+V u_{0}^{2}-\lambda_{1}\left(\bar{a}_{\Omega}\right) a u_{0}^{2}\right) d x \\
& =B\left(u_{0}\right) \leq 0,
\end{aligned}
$$

this implies that $\int_{\Omega}\left(\left|\nabla u_{0}\right|^{2}-\lambda_{1}\left(\bar{a}_{\Omega}\right) \bar{a}_{\Omega} u_{0}^{2}\right) d x=0$ and we must have $u_{0}=k \phi_{1}$ for some $k$. But, as $B\left(\phi_{1}\right)<0$, it follows that $k=0$. Therefore, $u_{n} \rightarrow 0$ in $X$. Next, let $v_{n}=\frac{u_{n}}{\left\|u_{n}\right\|_{\mu_{n}}}$. Then by Lemma 2.4, we may assume that there exists $v_{0} \in H_{0}^{1}(\Omega) \backslash\{0\}$ such that $v_{n} \rightarrow v_{0}$ in $X, v_{n} \rightarrow v_{0}$ in $L^{r}\left(\mathbb{R}^{N}\right)$ for all $2 \leq r<2^{\star}$ and $B\left(v_{n}\right) \rightarrow B\left(v_{0}\right)$. Thus,

$$
\lim _{n \rightarrow \infty} \int_{\mathbb{R}^{N}} a v_{n}^{2} d x=\int_{\mathbb{R}^{N}} a v_{0}^{2} d x
$$

Clearly,

$$
\left\|v_{n}\right\|^{2}-\lambda_{n} \int_{\mathbb{R}^{N}} a v_{n}^{2} d x \leq A_{\mu_{n}, \lambda_{n}}\left(v_{n}\right)=\left\|u_{n}\right\|_{\mu_{n}}^{2 p-2} B\left(v_{n}\right)<0
$$

for $n$ sufficiently large. We now show that $v_{n} \rightarrow v_{0}$ in $X$. Suppose on the contrary. Then by (4.34) and (4.35),

$$
\begin{aligned}
\int_{\Omega}\left(\left|\nabla v_{0}\right|^{2}-\lambda_{1}\left(\bar{a}_{\Omega}\right) \bar{a}_{\Omega} v_{0}^{2}\right) d x & =\int_{\mathbb{R}^{N}}\left(\left|\nabla u_{0}\right|^{2}+V v_{0}^{2}-\lambda_{1}\left(\bar{a}_{\Omega}\right) \bar{a}_{\Omega} v_{0}^{2}\right) d x \\
& <\liminf _{n \rightarrow \infty}\left(\left\|v_{n}\right\|^{2}-\lambda_{n} \int_{\mathbb{R}^{N}} a v_{n}^{2} d x\right) \leq 0,
\end{aligned}
$$

and so

$$
\int_{\Omega}\left(\left|\nabla v_{0}\right|^{2}-\lambda_{1}\left(\bar{a}_{\Omega}\right) \bar{a}_{\Omega} v_{0}^{2}\right) d x<0,
$$

which gives a contradiction. Hence $v_{n} \rightarrow v_{0}$ in $X$, which indicates that $\int_{\Omega}\left|\nabla v_{0}\right|^{2} d x=1$ and

$$
\int_{\Omega}\left(\left|\nabla v_{0}\right|^{2}-\lambda_{1}\left(\bar{a}_{\Omega}\right) \bar{a}_{\Omega} v_{0}^{2}\right) d x=0
$$

Therefore, $v_{0}=\phi_{1}$.

(ii) For any sequence $\mu_{n} \rightarrow \infty$, let $u_{n}^{(j)}:=u_{\mu_{n}}^{(j)}(j=1,2)$ be the solutions obtained in Theorem 1.3 with $u_{\mu_{n}}^{(1)} \in$ 
$\mathbf{N}_{\mu_{n}, \lambda}^{+}$and $u_{\mu_{n}}^{(2)} \in \mathbf{N}_{\mu_{n}, \lambda}^{-}$. Similar to the argument of proofs in Lemma 4.4 (ii) and Lemma 4.2 (i) there exists a positive constant $c_{0}$ is independent of $\mu_{n}$ such that

$$
\left\|u_{n}^{(j)}\right\|_{\mu_{n}} \leq c_{0} .
$$

Therefore, by Lemma 2.4, we may assume that there exist $u_{0}^{(j)} \in H_{0}^{1}(\Omega)$ such that $u_{n}^{(j)} \rightarrow u_{0}^{(j)}$ in $X$ and $B\left(u_{n}^{(j)}\right) \rightarrow B\left(u_{0}^{(j)}\right)$. Now for any $\varphi \in C_{0}^{\infty}(\Omega)$, because $\left\langle J_{\mu_{n}, \lambda}^{\prime}\left(u_{n}^{(j)}\right), \varphi\right\rangle=0$, it is easy to check that

$$
\begin{aligned}
& \int_{\Omega} \nabla u_{0}^{(j)} \nabla \varphi d x-\lambda \int_{\Omega} \bar{a}_{\Omega} u_{0}^{(j)} \varphi d x+\int_{\Omega}\left(I_{\alpha} \star\left|u_{0}^{(j)}\right|^{p}\right)\left|u_{0}^{(j)}\right|^{p-2} u_{0}^{(j)} \varphi d x \\
= & \int_{\Omega} f\left|u_{0}^{(j)}\right|^{2 p-2} u_{0}^{(j)} \varphi d x,
\end{aligned}
$$

that is, $u_{0}^{(j)}$ are weak solutions of Eq. $\left(P_{\infty}\right)$ by the density of $C_{0}^{\infty}(\Omega)$ in $H_{0}^{1}(\Omega)$. Now, we show that $u_{n}^{(j)} \rightarrow u_{0}^{(j)}$ in $X$ for $j=1$, 2. Because $\left\langle J_{\mu_{n}, \lambda}^{\prime}\left(u_{n}^{(j)}\right), u_{n}^{(j)}\right\rangle=\left\langle J_{\mu_{n}, \lambda}^{\prime}\left(u_{n}^{(j)}\right), u_{0}^{(j)}\right\rangle=0$, we have

$$
\left\|u_{n}^{(j)}\right\|_{\mu_{n}}^{2}-\lambda \int_{\mathbb{R}^{N}} a\left(u_{n}^{(j)}\right)^{2} d x+\int_{\Omega}\left(I_{\alpha} \star\left|u_{n}^{(j)}\right|^{p}\right)\left|u_{n}^{(j)}\right|^{p} d x=\int_{\mathbb{R}^{N}} f\left|u_{n}^{(j)}\right|^{p} d x
$$

and

$$
\begin{aligned}
& \left\langle u_{n}^{(j)}, u_{0}^{(j)}\right\rangle_{\mu_{n}}-\lambda \int_{\mathbb{R}^{N}} a u_{n}^{(j)} u_{0}^{(j)} d x+\int_{\Omega}\left(I_{\alpha} \star\left|u_{n}^{(j)}\right|^{p}\right)\left|u_{n}^{(j)}\right|^{p-2} u_{n}^{(j)} u_{0}^{(j)} d x \\
= & \int_{\mathbb{R}^{N}} f\left|u_{n}^{(j)}\right|^{p-2} u_{n}^{(j)} u_{0}^{(j)} d x .
\end{aligned}
$$

By (4.36) - (4.38) and $u_{n}^{(j)} \rightarrow u_{0}^{(j)}$ in $L^{r}\left(\mathbb{R}^{N}\right)$ for all $2 \leq r<2^{\star}$, we have

$$
\lim _{n \rightarrow \infty}\left\|u_{n}^{(j)}\right\|_{\mu_{n}}^{2}=\lim _{n \rightarrow \infty}\left\langle u_{n}^{(j)}, u_{0}^{(j)}\right\rangle_{\mu_{n}}=\lim _{n \rightarrow \infty}\left\langle u_{n}^{(j)}, u_{0}^{(j)}\right\rangle=\left\|u_{0}^{(j)}\right\|^{2}
$$

On the other hand, the weakly lower semi-continuity of norm yields

$$
\left\|u_{0}^{(j)}\right\|^{2} \leq \liminf _{n \rightarrow \infty}\left\|u_{n}^{(j)}\right\|^{2} \leq \lim _{n \rightarrow \infty}\left\|u_{n}^{(j)}\right\|_{\mu_{n}}^{2}
$$

and thus, $u_{n}^{(j)} \rightarrow u_{0}^{(j)}$ in $X$ for $j=1$, 2. By Lemma $4.4(i)$ and the fact that $\left\|u_{n}^{(2)}\right\|_{\mu_{n}}>c_{0}>0$, which implies that $u_{0}^{(2)} \neq 0$. Moreover, by (4.8), there exists $\kappa_{0}<0$

$$
J_{\mu_{n}, \lambda}\left(u_{n}^{(1)}\right)=\inf _{u \in \mathbf{N}_{\mu_{n}, \lambda}^{+}} J_{\mu_{n}, \lambda}(u) \leq \kappa_{0} \text { for all } n
$$

Thus,

$$
\left.J_{\mu, \lambda}\right|_{H_{0}^{1}(\Omega)}\left(u_{0}^{(1)}\right) \leq \kappa_{0}<0,
$$

which implies that $u_{0}^{(1)} \neq 0$. To complete the proof, it remains to show that $u_{0}^{(1)}$ and $u_{0}^{(2)}$ are distinct. That $\left.J_{\mu, \lambda}\right|_{H_{0}^{1}(\Omega)}\left(u_{0}^{(1)}\right) \leq \kappa_{0}<0$ and $\left.J_{\mu, \lambda}\right|_{H_{0}^{1}(\Omega)}\left(u_{0}^{(2)}\right)>0$ implies that $u_{0}^{(1)} \neq u_{0}^{(2)}$. This completes the proof.

Acknowledgement: This research was supported in part by the Ministry of Science and Technology, Taiwan (Grant No. 106-2115-M-390-002-MY2) and the National Center for Theoretical Sciences, Taiwan. 


\section{References}

[1] H. Amann and J. Lopez-Gomez, A priori bounds and multiple solutions for superlinear indefinite elliptic problems, J. Differential Equations 146, (1998), 336-374.

[2] A. Azzollini, Concentration and compactness in nonlinear Schrödinger-Poisson system with a general nonlinearity, J. Differential Equations 249, (2010), 1746-1763.

[3] C. O. Alves and A. B. Nobrega, Multi-bump solutions for Choquard equation with deepening potential well, Calc. Var. Partial Diff. Equ. (2016), 55:48.

[4] A. Ambrosetti and D. Ruiz, Multiple bound states for the Schrödinger-Poisson problem, Commum. Contemp. Math. 10 (2008), 391-404.

[5] P.A. Binding, P. Drabek, Y.X. Huang, On Neumann boundary value problems for some quasilinear elliptic equations, Electron. J. Differential Equations 5, (1997), 1-11.

[6] P.A. Binding, P. Drabek, Y.X. Huang, Existence of multiple solutions of critical quasilinear elliptic Neumann problems, Nonlinear Anal. 42, (2000), 613-629.

[7] V. Benci and D. Fortunato, An eigenvalue problem for the Schrödinger-Maxwell equations, Topol. Methods Nonlinear Anal. 11, (1998), no. 2, 283-293.

[8] H. Brezis and E. Lieb, A relation between point convergence of functions and convergence of functionals, Proc. Amer. Math. Soc. 88, (1983), 486-490.

[9] T. Bartsch, A. Pankov, Z. Q. Wang, Nonlinear Schrödinger equations with steep potential well, Commun. Contemp. Math. 3, (2001), 549-569.

[10] T. Bartsch and Z. Tang, Multibump solutions of nonlinear Schrödinger equations with steep potential well and indefinite potential, Discr. Cont. Dyna. Systems A 33, (2013), 7-26.

[11] T. Bartsch and Z. Q. Wang, Existence and multiplicity results for superlinear elliptic problems on $\mathbb{R}^{N}$, Comm. Partial Differential Equations 20, (1995), 1725-1741.

[12] K.J. Brown and Y. Zhang, The Nehari manifold for a semilinear elliptic equation with a sign-changing weight function, J. Differential Equations 193, (2003), 481-499.

[13] J. Chabrowski and D.G. Costa, On a class of Schrödinger-Type equations with indefinite weight functions, Comm. Partial Differential Equations 33, (2008), 1368-1394.

[14] J. Chen, Multiple positive solutions of a class of non autonomous Schrödinger-Poisson systems, Nonlinear Analysis: Real World Appl. 21, (2015), 13-26.

[15] D.G. Costa and H. Tehrani, Existence of positive solutions for a class of indefinite elliptic problems in $\mathbb{R}^{N}$, Calc. Var. Partial Diff. Equ. 13, (2001), 159-189.

[16] G. Cerami and G. Vaira, Positive solutions for some non-autonomous Schrödinger-Poisson systems, J. Differential Equations 248, (2010), 521-543.

[17] P. Drábek and S.I. Pohozaev, Positive solutions for the $p$-Laplacian: application of the fibrering method, Proc. Roy. Soc. Edinburgh A 127, (1997), 703-726.

[18] Y. Deng and W. Shuai, Sign-changing multi-bump solutions for Kirchhoff-type equations in $\mathbb{R}^{N}$, Discr. Cont. Dyna. Systems A 38, (2018), 3139-3168.

[19] I. Ekeland, Convexity methods in Hamiltonian mechanics, Springer, 1990.

[20] R.L. Frank and E.H. Lieb, Inversion positivity and the sharp Hardy-Littlewood-Sobolev inequality, Calc. Var. Partial Differ. Equ. 39, (2010), 85-99.

[21] L. Huang, E. M. Rocha, J. Chen, Two positive solutions of a class of Schrödinger-Poisson system with indefinite nonlinearity, J. Differential Equations 255, (2013), 2463-2483.

[22] L. Huang, E.M. Rocha, J. Chen, On the Schrödinger-Poisson system with a general indefinite nonlinearity, Nonlinear Analysis: Real World Appl. 28, (2016), 1-19.

[23] I. Ianni and G. Vaira, On concentration of positive bound states for the Schrödinger-Poisson problems with potentials, Adv. Nonlinear Stud. 8, (2008), 573-595.

[24] Y. Jiang and H. Zhou, Schrödinger-Poisson system with steep potential well, J. Differential Equations 251, (2011), $582-608$.

[25] E.H. Lieb, Sharp constants in the Hardy-Littlewood-Sobolev and related inequalities. Ann. Math. 118, (1983), 349-374.

[26] E.H. Lieb and M. Loss, Analysis, 2nd ed., Graduate Studies in Math. 14, American Mathematical Society, Providence, 2001.

[27] P.L. Lions, The concentration-compactness principle in the calculus of variations. The locally compact case, Part I, Ann. Inst. H. Poincare Anal. Non Lineaire 1, (1984), 109-145.

[28] V. Moroz and J. Van Schaftingen, Groundstates of nonlinear Choquard equations: Existence, qualitative properties and decay asymptotics, J. Funct. Anal. 265, (2013), 153-184.

[29] Z. Nehari, On a class of nonlinear second-order differential equations, Trans. Amer. Math. Soc. 95, (1960), 101-123.

[30] D. Ruiz, The Schrödinger-Poisson equation under the effect of a nonlinear local term, J. Funct. Anal. 237, (2006), 655-674.

[31] D. Ruiz, On the Schrödinger-Poisson-Slater system: behavior of minimizer, radial and nonradial cases, Arch. Ration. Mech. Anal. 198, (2010), 349-368. 
[32] Z. Shen and Z. Han, Multiple solutions for a class of Schrödinger-Poisson system with indefinite nonlinearity, J. Math.Anal.Appl. 426, (2015), 839-854.

[33] J. Sun and T.F. Wu, Ground state solutions for an indefinite Kirchhoff type problem with steep potential well, J. Differential Equations 256, (2014), 1771-1792.

[34] J. Sun and T.F. Wu, On the nonlinear Schrödinger-Poisson systems with sign-changing potential, Z. Angew. Math. Phys. 66, (2015), 1649-1669.

[35] J. Sun, T.F. Wu, Z. Feng, Multiplicity of positive solutions for a nonlinear Schrödinger-Poisson system, J. Differential Equations 260, (2016), 586-627.

[36] J. Sun, T.F. Wu, Z. Feng, On the non-autonomous Schrödinger-Poisson problems in $\mathbb{R}^{3}$, Discrete Contin. Dyn. Syst. A 38, (2018), 1889-1933.

[37] J. Sun, T.F. Wu, Y. Wu, Existence of nontrivial solution for Schrödinger-Poisson systems with indefinite steep potential well, Z. Angew. Math. Phys. 68, (2017), 1-22.

[38] C. Stuart and H. Zhou, Global branch of solutions for nonlinear Schrödinger equations with deepening potential well, Proc. Lond. Math. Soc. 92, (2006), 655-681.

[39] N. S. Trudinger, On Harnack type inequalities and their application to quasilinear elliptic equations, Comm. Pure Appl. Math. 20, (1967), 721-747.

[40] G. Vaira, Ground states for Schrödinger-Poisson type systems. Ric. Mat. 60, (2011), 263-297.

[41] G. Vaira, Existence of bound states for Schrödinger-Newton type systems. Adv. Nonlinear Stud. 13, (2013), $495-516$.

[42] M. Willem, Minimax Theorems, Birkhäuser, Boston, 1996.

[43] Z. Wang and H. Zhou, Positive solutions for nonlinear Schrödinger equations with deepening potential well, J. Europ. Math. Soc. 11, (2009), 545-573.

[44] T. F. Wu, The Nehari manifold for indefinite nonlinear Schrödinger equations in $\mathbb{R}^{N}$, preprint.

[45] L. Zhao, H. Liu, F. Zhao, Existence and concentration of solutions for the Schrödinger-Poisson equations with steep well potential, J. Differential Equations 255, (2013), 1-23.

[46] X. Zhang and S. Ma, Multi-bump solutions of Schrödinger-Poisson equations with steep potential well, Z. Angew. Math. Phys. 66, (2015), 1615-1631. 